\title{
Ion motion in the current sheet with sheared magnetic field - Part 1: Quasi-adiabatic theory
}

\author{
A. V. Artemyev ${ }^{1,2}$, A. I. Neishtadt ${ }^{1,3}$, and L. M. Zelenyi ${ }^{1}$ \\ ${ }^{1}$ Space Research Institute, RAS, Profsouznaya st., 84/32, GSP-7, 117997 Moscow, Russia \\ ${ }^{2}$ Laboratoire de physique et chimie de l'environnement et de l'Espace (LPC2E), UMR7328, \\ CNRS - Université d'Orléans, 3A, Avenue de la Recherche Scientifique, 45071 Orleans cedex 2, France \\ ${ }^{3}$ Department of Mathematical Sciences, Loughborough University, Loughborough LE11 3TU, UK
}

Correspondence to: A. V. Artemyev (ante0226@gmail.com)

Received: 2 June 2012 - Revised: 18 January 2013 - Accepted: 21 January 2013 - Published: 21 February 2013

\begin{abstract}
We present a theory of trapped ion motion in the magnetotail current sheet with a constant dawn-dusk component of the magnetic field. Particle trajectories are described analytically using the quasi-adiabatic invariant corresponding to averaging of fast oscillations around the tangential component of the magnetic field. We consider particle dynamics in the quasi-adiabatic approximation and demonstrate that the principal role is played by large (so called geometrical) jumps of the quasi-adiabatic invariant. These jumps appear due to the current sheet asymmetry related to the presence of the dawn-dusk magnetic field. The analytical description is compared with results of numerical integration. We show that there are four possible regimes of particle motion. Each regime is characterized by certain ranges of values of the dawn-dusk magnetic field and particle energy. We find the critical value of the dawn-dusk magnetic field, where jumps of the quasi-adiabatic invariant vanish.
\end{abstract}

\section{Introduction}

Description of charged-particle dynamics in strongly inhomogeneous magnetic fields with a small curvature radius is an important problem of plasma physics. This description has applications in theory of thin current sheets in the Earth's magnetotail (see reviews by Zelenyi et al., 2011; Birn et al., 2012, and references therein), magnetopause (see discussion in Whipple et al., 1984; Panov et al., 2011), and for many other mesoscale structures observed in the magnetosphere (see review by Sharma et al., 2008; Grigorenko et al., 2011, and references therein) and solar corona (e.g.
Litvinenko, 1993; Anastasiadis et al., 2008 and references therein). Moreover, models of penetration of solar wind particles into magnetosphere (Zhou et al., 2007) and electron dynamics in the radiation belts (see e.g. Ukhorskiy et al., 2011, and references therein) also have a relation to this topic.

In a general situation, to solve Hamiltonian equations of charged particle motion analytically, one needs to introduce additional invariants besides the well-known energy and momentum. One of the most effective theories developed in this way is the guiding-center theory of charged particle motion, where the magnetic moment can be introduced as this additional invariant of motion (Northrop, 1963; Sivukhin, 1965). This theory is valid for systems with strong magnetic fields, where spatial and temporal scales of magnetic field variations are much larger and slower than typical scales of particle motion.

The guiding-center theory in a general case cannot be applied to describe ion dynamics in the Earth's magnetotail with weak magnetic field in the vicinity of the current sheet. However, in such systems, another small parameter can be introduced. This parameter is defined as the ratio of the curvature radius of field lines and ion gyroradius. Thus, complementary adiabatic type theory of particle motion can be developed in a more general form. Such a new theory is based on the adiabatic invariance of the integral of action (Landau and Lifshitz, 1960; Schindler, 1965; Sonnerup, 1971; Whipple et al., 1986). This invariant is often called quasi-adiabatic invariant to distinguish it from the magnetic moment. Usually quasi-adiabatic invariants are more poorly conserved in comparison with the magnetic moment. 
Ion trajectories in the current sheet can be described analytically using the quasi-adiabatic invariant (Büchner and Zelenyi, 1986, 1989; Chen, 1992). This description was also generalized to manage current sheets with the X-line and the dawn-dusk electric field (Vainchtein et al., 2005) or bifurcated current sheets (Artemyev et al., 2011; Vasiliev et al., 2012). The description of particle trajectories is based on separation of the particle motion into two components: fast oscillations around field lines (or across the current sheet) and slow oscillations along field lines (or rotation in the current sheet plane). Roughly speaking, averaging over fast oscillations gives the quasi-adiabatic invariant.

Systems under consideration contain the separatrix in the phase plane of fast variables. Trajectories cross the separatrix when particles change the character of their motion from motion along field lines far from the current sheet plane to rotation in (and oscillations across) the current sheet plane. Crossings of the separatrix result in the violation of the conservation of the quasi-adiabatic invariant: so-called jumps (Timofeev, 1978; Neishtadt, 1986; Cary et al., 1986; Büchner and Zelenyi, 1989).

Previously, the theory of the quasi-adiabatic invariants and related ion motion in the magnetotail current sheet was developed for systems without shear of the magnetic field, i.e. with zero value of the dawn-dusk component $B_{y}$ of the magnetic field (hereafter we use GSM coordinate system). Only the tangential component $B_{x}(z)$ and the normal component $B_{z}$ were taken into account. However, $B_{y}$ component is often present in the magnetotail (see Petrukovich, 2011, and references therein). It plays an important role in the current sheet formation (see Silin and Büchner, 2006; Artemyev, 2011; Malova et al., 2012, and references therein) and stability (see Galeev et al., 1986; Kuznetsova et al., 1996; Silin and Büchner, 2003; Karimabadi et al., 2005, and references therein).

There are several numerical investigations of the influence of the magnetic field shear $\left(B_{y} \neq 0\right)$ on ion motion (Karimabadi et al., 1990; Büchner and Zelenyi, 1991; Zhu and Parks, 1993; Kaufmann et al., 1994; Baek et al., 1995; Holland et al., 1996; Chapman and Rowlands, 1998; Delcourt et al., 2000; Ynnerman et al., 2000). Major parts of these numerical investigations are devoted to the description of peculiarities of particle motion for particular values of system parameters. However, analytical theory of this motion is still not properly developed. In this paper we develop an adiabatic theory of ion motion in the current sheet with $B_{y} \neq 0$. In the discussion we compare main results of previous numerical investigations with our analytical theory.

In this paper we only consider motion of trapped particles (this is the first part of our investigation). Moreover, we take into account only adiabatic motion without effects of stochastic destruction of the quasi-adiabatic invariant. The non-adiabatic effects (including peculiarities of untrapped motion) will be considered in the next paper.

\section{General equations}

A simple model of the magnetic field component $B_{x}$ in the current sheet can be represented as $B_{x}=B_{0}(z / L)$ for $|z / L|<1$ and $B_{x}= \pm B_{0}$ for $|z / L|>1$, where $L$ is the current sheet thickness and $B_{0}$ is the amplitude of $B_{x}$. In the system without $B_{y}$, the field lines have the shape of parabolas $B_{z} x=B_{0} z^{2} / 2 L+$ const (inside the domain $|z / L|<1$ ). Particle motion can be qualitatively described as follows: far from the neutral plane $z=0$ (where $B_{x}=0$ ) particles quickly rotate around the field lines and move along them. When particles approach the neutral plane $z=0$, they change the type of motion: now, instead of rotation around field lines, particles rotate around $B_{z}$ and oscillate across the neutral plane $z=0$. After half a period of the rotation around $B_{z}$, particles leave the neutral plane $z=0$ and return to the motion along field lines. This type of trajectory was discovered by Speiser (see Speiser, 1965, 1967). The description of this motion in the system with $B_{y}=0$ was given by Sonnerup (1971); Chen and Palmadesso (1986); Büchner and Zelenyi (1986, 1989). Far from the neutral plane $z=0$, due to the growth of $B_{x}(z)$ component, particles can make a turn and start moving towards the neutral plane again. This motion is bounded and can be considered as trapped. If particles reach the current sheet boundary where $B_{x}$ component becomes constant $\left(B_{x}= \pm B_{0}\right)$, the motion becomes unbounded and can be considered as transient one. Corresponding particle trajectories are open. In this paper we are interested mainly in particle behaviour in the vicinity of the neutral plane $z=0$ during periodical returns of the particle's trajectory to this region. Thus, we consider the trapped motion. Effect of open trajectories and related particle losses is a subject of the next publication.

We start with brief discussion of main features of trapped trajectories in current sheets with $B_{y}=0$. For this purpose we show particle trajectories in the current sheet without $B_{y}$ (Fig. 1a, b). In case of $B_{y}=0$ each particle can move along one of two possible segments of a trajectory: with a turnpoint at positive $z$ (Fig. 1a) and at negative $z$ (Fig. 1b). Projections of these two segments onto the neutral plane $z=0$ coincide. Both segments consist of two parts: rotation around $B_{z}$ in the neutral plane $z=0$ (the corresponding oscillations over $\mathrm{Z}$-coordinate have a large amplitude) and motion along field lines (the amplitude of the oscillations around field lines is about two times smaller). Any particle moving along one of the segments can change it to another one at the moment of leaving the neutral plane $z=0$ (when the amplitude of $z$ oscillations decreases twice). Detailed description of such trajectories can be found in Büchner and Zelenyi (1986, 1989).

In the case of $B_{y} \neq 0$, particle trajectories are more complicated (Fig. 1c). Both previous segments merge into a single trajectory. This trajectory consists of four segments. The two segments A1 and A2 correspond to the motion along field lines and have turning points at positive and negative $z$ respectively. Their projections onto the neutral plane $z=0$ 

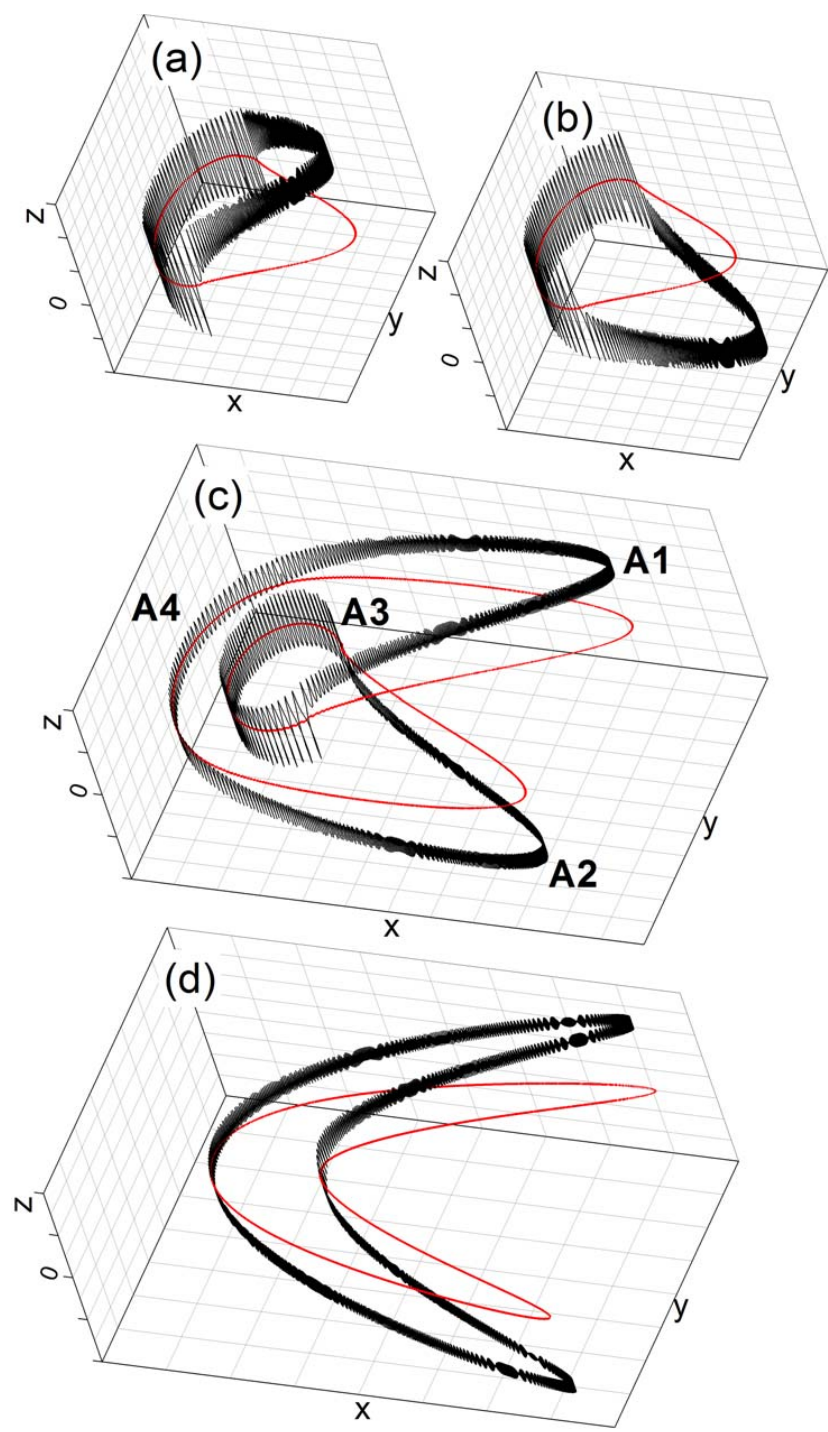

Fig. 1. Characteristic trajectories in current sheets without $B_{y}$ (panels $\mathbf{a}$ and $\mathbf{b}, s=0$ and $\kappa=0.01$ ) and with $B_{y}$ (panel $\mathbf{c}$ corresponds to $s=0.5$ and $\kappa=0.01$ and panel $\mathbf{d}$ corresponds to $s=1.0$ and $\kappa=0.01)$. Red curves show projection of trajectories onto plane $z=0$.

do not coincide. The segment A3 with the increased amplitude of z-oscillations corresponds to rotation around $B_{z}$ in the neutral plane $z=0$. The segment A4 corresponds to rotation around $B_{z}$ in the vicinity of the neutral plane $z=0$ without the increase of the amplitude of z-oscillations. Note that if the value of $B_{y}$ is large enough, the segment A3 with large zoscillations and rotation around $B_{z}$ in the neutral plane $z=0$ disappears (Fig. 1d). This complex motion of a charged particle in current sheets with various values of $B_{y}$ is the subject of our study. Without loss of generality we consider positive $B_{y}$ (negative $B_{y}$ corresponds to the mirror reflection of the system with respect to the neutral plane $z=0$ ).
We study dynamics of particles in the system with the magnetic field $\boldsymbol{B}=B_{0}(z / L) \boldsymbol{e}_{x}+B_{y} \boldsymbol{e}_{y}+B_{z} \boldsymbol{e}_{z}$, where $B_{z}>0$ and $B_{y}>0$ are constants. Corresponding vector potential is $\boldsymbol{A}=B_{y} z \boldsymbol{e}_{x}+\left(B_{z} x-B_{0} z^{2} / 2 L\right) \boldsymbol{e}_{y}$. The Hamiltonian of a particle with mass $m$ and charge $q$ in this system has the form

$H=\frac{1}{2 m} p_{z}^{2}+\frac{1}{2 m}\left(p_{x}-\frac{q}{c} B_{y} z\right)^{2}+\frac{1}{2 m}\left(p_{y}-\frac{q}{c} B_{z} x+\frac{q}{2 c L} B_{0} z^{2}\right)^{2}$.

We note that particle energy is constant $H=h=$ const because Hamiltonian does not depend on time explicitly $(\partial H / \partial t=0)$. We use dimensionless variables $\boldsymbol{p} \rightarrow \boldsymbol{p} / \sqrt{2 h m}, \boldsymbol{r} \rightarrow \boldsymbol{r} / \sqrt{\rho_{0} L}$, dimensionless time $t \rightarrow$ $t \sqrt{2 h /\left(\rho_{0} L m\right)}$, parameters $\kappa=B_{z} / B_{0} \sqrt{L / \rho_{0}}$ and $s=$ $B_{y} / B_{0} \sqrt{L / \rho_{0}}\left(\rho_{0}=\sqrt{2 h m} c /\left(q B_{0}\right)\right.$ is the Larmor radius $)$. We also shift coordinate system along $x$ to set momentum $p_{y}=0$ (it is possible because the Hamiltonian does not depend on y-coordinate). In this case, dimensionless Hamiltonian $H \rightarrow H / 2 h$ can be written as

$H=\frac{1}{2} p_{z}^{2}+\frac{1}{2}\left(p_{x}-s z\right)^{2}+\frac{1}{2}\left(\kappa x-\frac{1}{2} z^{2}\right)^{2}$.

Our normalization of all variables on particle energy $h$ gives the equation $H\left(z, p_{z}, \kappa x, p_{x}\right)=1 / 2$. Thus, in the fourdimensional space $\left(z, p_{z}, \kappa x, p_{x}\right)$ the particle moves on the three-dimensional surface $H\left(z, p_{z}, \kappa x, p_{x}\right)=$ const. If $B_{y}=$ 0 , we have $s=0$ and Hamiltonian (1) is the same as the one considered earlier by Sonnerup (1971); Büchner and Zelenyi (1986, 1989). Parameter $\kappa$ is small for thin current sheets observed in the magnetotail $\left(\kappa \in[0.01,0.1]\right.$ where $L \sim \rho_{0}$; see review by Zelenyi et al., 2011 and references therein). Therefore, variables $\left(\kappa x, p_{x}\right)$ are slow and variables $\left(z, p_{z}\right)$ are fast.

\section{Fast variables and quasi-adiabatic invariant}

We introduce the potential energy $U\left(\kappa x, p_{x}, z\right)=H-\frac{1}{2} p_{z}^{2}$ of particle motion in the phase plane $\left(z, p_{z}\right)$ of fast variables. At given values of the slow variables, system (1) could be considered as a Hamiltonian system with one degree of freedom with $H=\frac{1}{2} p_{z}^{2}+U\left(\kappa x, p_{x}, z\right)$, where $\left(\kappa x, p_{x}\right)$ are treated as constant parameters.

Function $U=U\left(z, \kappa x, p_{x}\right)$ is the fourth order polynomial. The particle's oscillations in the potential $U$ occur on the energy level $H=1 / 2$, i.e. amplitudes of these oscillations are determined by the equation $U=1 / 2$. Function $U\left(z, \kappa x, p_{x}\right)$ can have a single minimum or two minima separated by a local maximum depending on values of $\kappa x, p_{x}$. Thus, the equation $U=1 / 2$ can have two solutions or four solutions. Here we do not consider such values of $\kappa x, p_{x}$ that the equation $U=1 / 2$ does not have any real solutions. For values of $\kappa x, p_{x}$ such that there are four solutions of the equation $U=1 / 2$, the schematic view of $U$ as a function of $z$ is presented in Fig. 2a. There are two minima and one local maximum at $z=z_{\mathrm{c}}$. The phase portrait of this system is presented in Fig. 2c. A system with two solutions of the equation $U=1 / 2$ is shown in Fig. 2b, d. 


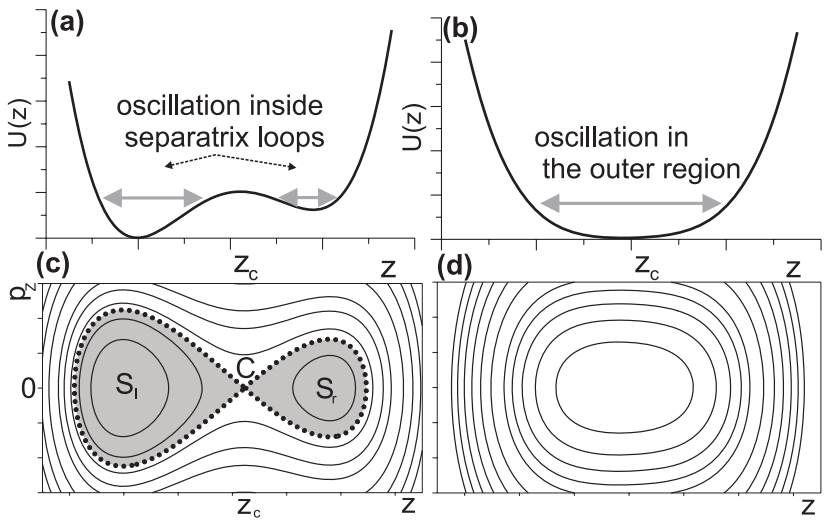

Fig. 2. Panels (a) and (b) demonstrate profile $U(z)$. Panels (c) and (d) demonstrate the corresponding phase plane of the fast variables. $S_{1}$ and $S_{\mathrm{r}}$ are the areas surrounded by separatrix loops.

One can see two types of particle trajectories in these portraits: the first type corresponds to particle oscillations inside one of the two small potential wells (these are particle oscillations around field lines, Fig. 2a, c). The second type corresponds to particle oscillations in the outer region with crossing $z=z_{\mathrm{c}}$ (Fig. 2a, c). Separatrix separates these two types of trajectories (it is shown by the dotted curve in Fig. 2c). Separatrix passes through the saddle point at $z=z_{\mathrm{c}}$. For the system with $B_{y}=0$, we have $z_{\mathrm{c}}=0$ and trajectories crossing $z=z_{\mathrm{c}}$ correspond to oscillations across the neutral plane $z=0$.

As particle trajectories in the plane $\left(z, p_{z}\right)$ are closed (i.e. motion is periodic), one can introduce the quasi-adiabatic invariant as the area surrounded by a trajectory divided by $2 \pi$ : $I_{z}=(1 / 2 \pi) \oint p_{z} d z$ (Landau and Lifshitz, 1960; Sonnerup, 1971; Büchner and Zelenyi, 1986).

\section{Slow variables}

Potential energy $U$ depends on the slow variables $\left(\kappa x, p_{x}\right)$, and we can divide the phase plane $\left(\kappa x, p_{x}\right)$ into several domains on the constant energy level $H=1 / 2$. Each domain corresponds to some profile of $U(z)$ and some position of the line $U=1 / 2$ with respect to this profile, see Fig. 3. Hereafter we use marks (t1), (t2l), (t2r) and (t2) for four domains with different types of position of the energy level $U=1 / 2$ relative to $U(z)$ profiles. Each domain is characterized by properties of solutions of the equation $U=1 / 2$. We consider location of these solutions with respect to the plane $z=0$ to determine particle position relative to the neutral plane. Note that for systems with $s \neq 0$, value $z=0$ does not play any particular role in the equation $U=1 / 2$ (for $s=0$ potential $U$ is symmetrical relative to the plane $z=0$ ). However, $z=0$ determines position of the neutral plane even for $s \neq 0$. Thus, it is important to describe particle motion relative to this plane.

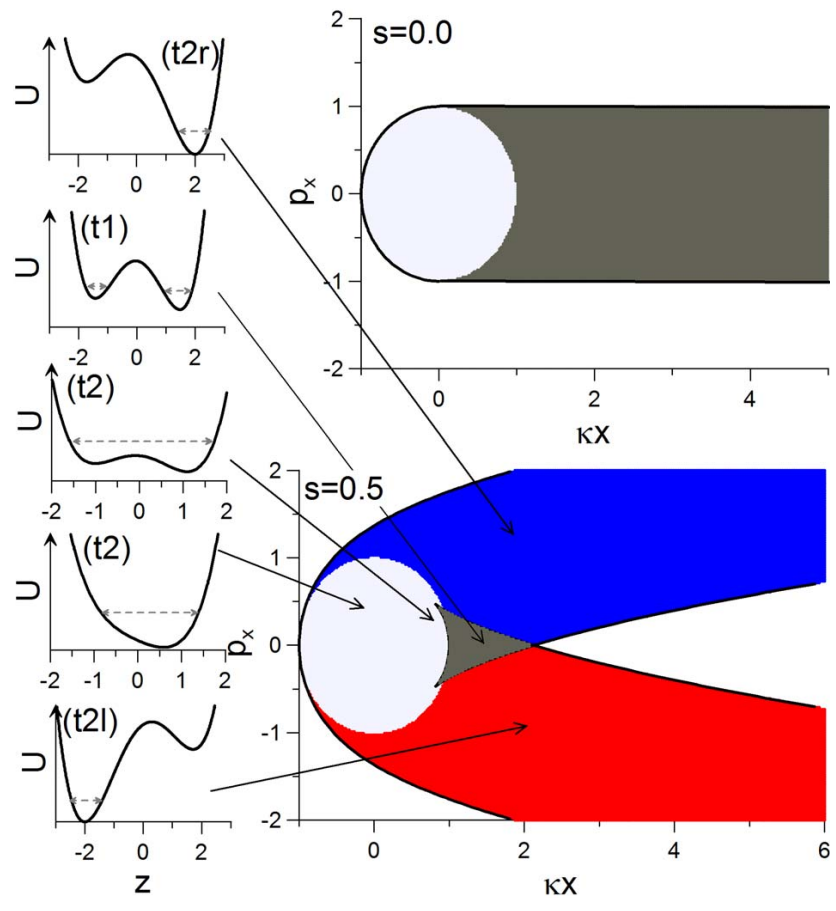

Fig. 3. The phase plane of slow variables $\left(\kappa x, p_{x}\right)$ is shown for two values of the parameter $s$. Various colours are used for domains with different types of particle motion. Dotted grey lines show the position of energy level $U=1 / 2$.

For the system with $s=0$ (i.e. $B_{y}=0$ ) we have only two domains. In the first domain (t1) particles oscillate in one of two symmetric potential wells. Thus, we have four solutions of the equation $U=1 / 2$. In the second domain (t2) particles oscillate in the single potential well (i.e. there is just one well, or there are two wells separated by the maximum of $U$ located below energy level $H=1 / 2$ ). In this domain we have two solutions of the equation $U=1 / 2$. When the particle moves inside the (t2) domain, it crosses the neutral plane $z=0$. Trajectories corresponding to oscillations in a single potential well (motion in the ( $\mathrm{t} 2$ ) domain) and in one of two potential wells (motion in the (t1) domain) are separated by the separatrix in the plane $\left(z, p_{z}\right)$ (see Fig. 2a, c). The separatrix corresponds to a certain curve in the $\left(\kappa x, p_{x}\right)$ plane. This curve is called the uncertainty curve. Thus, the uncertainty curve separates (t1) and (t2) domains in the plane $\left(\kappa x, p_{x}\right)$. When a particle is located on the uncertainty curve in the plane $\left(\kappa x, p_{x}\right)$, this particle moves along the separatrix in the $\left(z, p_{z}\right)$ plane. Hence, we use term the uncertainty curve on the phase plane $\left(\kappa x, p_{x}\right)$ and term the separatrix on the phase plane $\left(z, p_{z}\right)$ (the uncertainty curve was first introduced by Wisdom, 1985). For a system with $s=0$, the uncertainty curve is defined by the simple equation $(\kappa x)^{2}+p_{x}^{2}=1$, $\kappa x>0$.

If $s \neq 0$, two additional domains appear, while area of the domain (t1) with two potential wells decreases. These two new domains, (t2r) and (t2l,), correspond to particle 
oscillations inside a single potential well without crossing the neutral plane $z=0$. The particle oscillates above (blue colour) or below (red colour) the neutral plane $z=0$ inside the right or left potential wells, respectively. In this case the equation $U=1 / 2$ has two solutions. Both solutions are positive for (t2r) domain and negative for (t21) domain. For $s \neq 0$, the uncertainty curve is the boundary between (t1) and (t2) domains. Due to decrease of the (t1) domain area, the uncertainty curve shrinks. It looks like a segment of a circle.

At the boundaries between ( $\mathrm{t} 1)$ domain and $(\mathrm{t} 2 \mathrm{r}),(\mathrm{t} 2 \mathrm{l})$ domains, the bottom of one of the potential wells is located at the energy level $H=1 / 2$, but these boundaries are not uncertainty curves (crossing of these boundaries does not correspond to the separatrix crossing in the $\left(z, p_{z}\right)$ plane). The boundaries between ( $\mathrm{t} 2)$ domain and ( $\mathrm{t} 2 \mathrm{r}),(\mathrm{t} 2 \mathrm{l})$ domains are not an uncertainty curve either. These boundaries are defined by the following condition: one of solutions of the equation $U=1 / 2$ is equal to zero. This condition gives the equation of the boundary $(\kappa x)^{2}+p_{x}^{2}=1$. Figure 3 shows that if $s \neq 0$, the circle $(\kappa x)^{2}+p_{x}^{2}=1$ does not coincide with the uncertainty curve. Moreover, some points of the (t1) domain are located inside this circle. Thus, particles can cross the neutral plane $z=0$ in course of oscillations inside one of the two potential wells.

Due to evolution of slow variables, particles can go from one domain to another in the plane $\left(\kappa x, p_{x}\right)$ and cross the uncertainty curve (and hence cross the separatrix in the fast variable plane). Each transition from one domain to another corresponds to change in type of the particle's motion. Transition from the (t1) domain to the (t2) domain means that particles change oscillations around field lines to oscillations across the neutral plane $z=0$. Transition from the (t2) domain to the (t2l) (or (t2r)) domain means that particles change oscillations across the neutral plane to rotation around field lines below (or above) the neutral plane. Particles in ( $\mathrm{t} 2 \mathrm{l})$ and (t2l) domains do not cross the neutral plane $z=0$.

Any separatrix crossing (i.e. transitions from the (t1) domain to the ( $\mathrm{t} 2)$ domain and vice versa) results in a variation of $I_{z}$ (the so-called jump of the quasi-adiabatic invariant; see Timofeev, 1978; Cary et al., 1986; Neishtadt, 1986, 1987). A jump of the quasi-adiabatic invariant $\Delta I_{z}$ consists of two parts. The first part corresponds to violation of adiabaticity of particle motion in vicinity of the saddle point $z=z_{\mathrm{c}}$ (this is a dynamical jump, $\left.\Delta I_{z}^{\mathrm{dyn}}\right)$. The second part corresponds to difference of the areas surrounded by the particle's trajectory inside one of the separatrix loops and in the outer region outside of these loops (this is a geometrical jump $\Delta I_{z}^{\text {geom }}$ ).

\section{Adiabatic descriptions}

Invariant $I_{z}$ is not an exact integral of motion and is conserved only approximately. However, variations of $I_{z}$ far from the separatrix are proportional to the small parameter $\kappa$. These variations can be reduced by introduction of an im- proved invariant (see details in Neishtadt, 1986; Arnold et al., 2006). For a system with a symmetrical phase portrait in the plane $\left(z, p_{z}\right)$ (i.e. for $s=0$ ), dynamical jumps of the quasiadiabatic invariant $\Delta I_{z}^{\mathrm{dyn}}$ at the separatrix are proportional to $\kappa$. For asymmetrical phase portraits (i.e. for $s \neq 0$ ), dynamical jumps of the quasi-adiabatic invariant $\Delta I_{z}^{\mathrm{dyn}}$ at the separatrix are proportional to $\kappa \ln \kappa$ (Cary et al., 1986; Neishtadt, 1986, 1987). In the first approximation, we can neglect variations of $I_{z}$ far from the separatrix and dynamical jumps $\Delta I_{z}^{\mathrm{dyn}}$ at the separatrix. Such an approximation is called the adiabatic approximation. According to this approximation, particle trajectories in the phase plane of slow variables $\left(\kappa x, p_{x}\right)$ are defined by the equation $I_{z}\left(\kappa x, p_{x}\right)=$ const up to reaching the uncertainty curve. In the course of motion in the plane $\left(\kappa x, p_{x}\right)$ of slow variables, particle trajectory in the plane $\left(z, p_{z}\right)$ evolves. However, the area surrounded by the trajectory in the plane $\left(z, p_{z}\right)$ is conserved (i.e. $I_{z}=$ const).

The areas $S_{1, \mathrm{r}}$ surrounded by separatrix loops (see Fig. 2) are functions of the slow variables $\left(\kappa x, p_{x}\right)$. Thus, $S_{1, \mathrm{r}}$ depends on the particle's position in the $\left(\kappa x, p_{x}\right)$ plane. Particle motion in the $\left(\kappa x, p_{x}\right)$ plane results in evolution of $S_{1, \mathrm{r}}$. Velocity of this evolution $\kappa \Theta_{1, \mathrm{r}}$ can be determined as $\kappa \Theta_{1, \mathrm{r}}=\mathrm{d} S_{1, \mathrm{r}} / \mathrm{d} t$ (see details in Appendix A and in Arnold et al., 2006).

In the system with $s=0$, we have only two domains in the plane $\left(\kappa x, p_{x}\right)$. Particles move in the (t1) domain inside one of separatrix loops (inside one of two potential wells) with invariant $I_{z}=$ const and cross the separatrix. This crossing occurs when the area surrounded by this loop decreases and becomes equal to $2 \pi I_{z}$. After separatrix crossing, particles are inside the single potential well in the (t2) domain, where the area surrounded by the trajectory is exactly two times larger than the area before the crossing (both separatrix loops are symmetric and $S_{1}=S_{\mathrm{r}}$ ). As a result, one can use factor $1 / 2$ to renormalize the invariant $I_{z}$. Namely, one can introduce the quasi-adiabatic invariant as $I_{z}=(1 / 2 \pi) \oint p_{z} \mathrm{~d} z$ in the (t1) domain and as $I_{z}=(1 / 4 \pi) \oint p_{z} \mathrm{~d} z$ in the (t2) domain. In this case invariant $I_{z}$ is constant along the entire particle trajectory. Thus, the equation $I_{z}\left(\kappa x, p_{x}\right)=$ const determines all particle trajectories in the slow variable plane $\left(\kappa x, p_{x}\right)$. This approach is used to describe particle motion after averaging over the fast variable for a system with $s=0$ (see, e.g. Büchner and Zelenyi, 1989; Vainchtein et al., 2005, and references therein).

One additional property of the system with $s=0$ is identity of the velocities $\kappa \Theta_{1, \mathrm{r}}$ of $S_{1, \mathrm{r}}$ evolution. When particles approach the separatrix inside one of the two potential wells, the areas surrounded by both separatrix loops decrease. Trajectories cross the uncertainty curve (and cross the separatrix in the phase plane of fast variables) and should appear inside the single potential well in the (t2) domain, because the capture into one of two small wells is impossible due to the decrease of corresponding areas $S_{1, \mathrm{r}}$. This transition is shown in Fig. 4 (top panel, separatrix crossing C1). When particles 


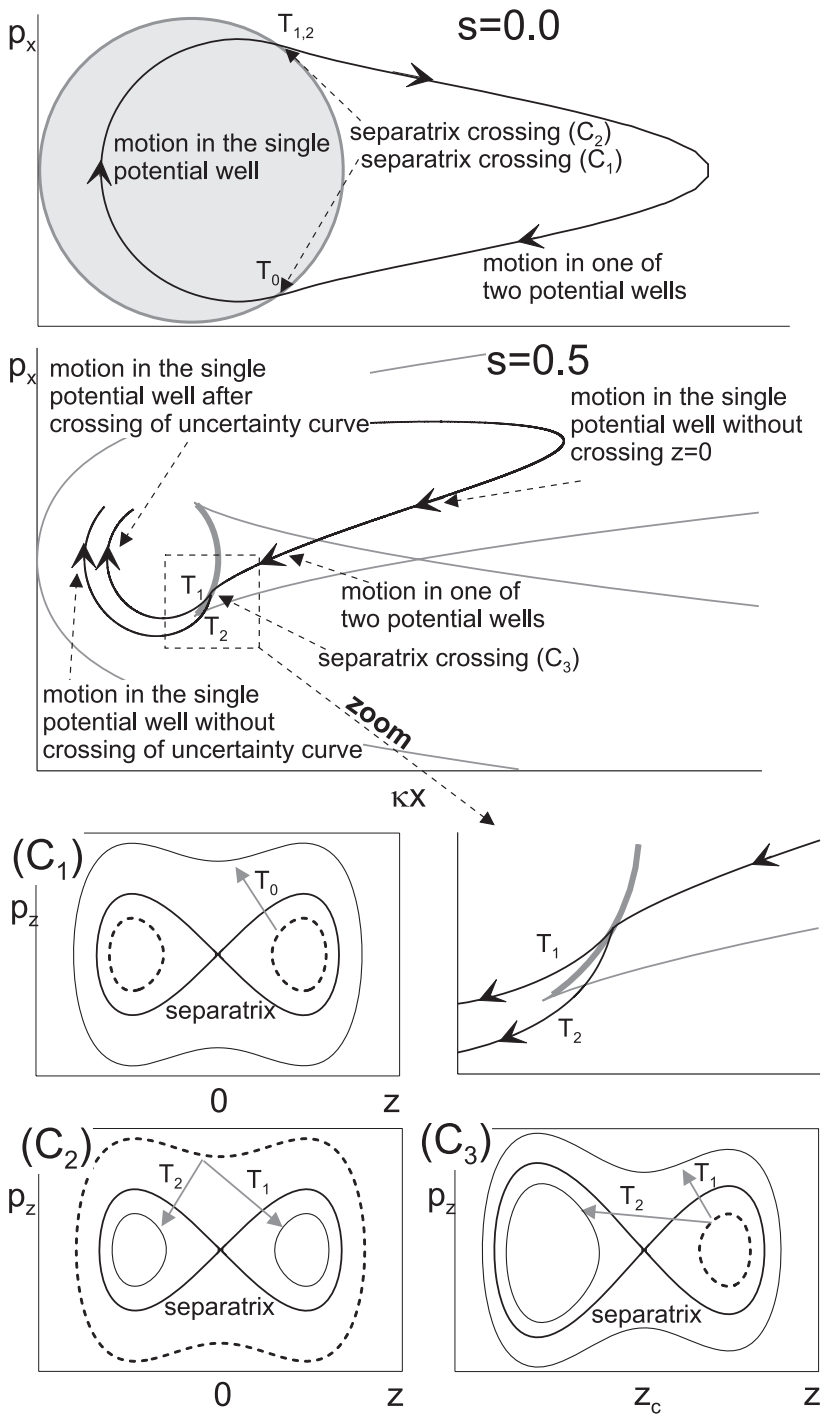

Fig. 4. Schemes of particle trajectories in systems with $s=0$ and with $s=0.5$ are shown in the phase plane $\left(\kappa x, p_{x}\right)$. Fragment of $\left(\kappa x, p_{x}\right)$ plane with trajectory splitting is shown in separated panel. Bottom schemes (C1, C2, C3) show particle trajectories before (dotted curves) and after (solid curves) separatrix crossings in the plane $\left(z, p_{z}\right)$.

oscillating in the single potential well in the (t2) domain approach the separatrix again, areas $S_{1, \mathrm{r}}$ increase. As a result, particles will be captured into one of the two potential wells in the (t1) domain. This transition is shown in Fig. 4 (top panel, separatrix crossing C2). Such a type of trapped motion is described in detail by Büchner and Zelenyi (1986, 1989); Vainchtein et al. (2005) (see also scheme in Fig. 4). In the adiabatic approximation, trapped-type particles move along a single trajectory in the phase plane $\left(\kappa x, p_{x}\right)$ forever.

In the system with $s \neq 0$, the areas of the two separatrix loops $S_{1, \mathrm{r}}$ are not equal. Moreover, the evolution of these areas is asynchronous (see Appendix A). As a result, we have two important new effects. Firstly, the quasi-adiabatic invariant cannot be conserved in the course of the separatrix crossings, even in the adiabatic approximation, because $S_{1} \neq S_{\mathrm{r}}$ (i.e. $S_{1}+S_{\mathrm{r}} \neq 2 S_{\mathrm{r}} \neq 2 S_{1}$ ) and there is no factor $1 / 2$ of renormalization (as it was for the case $s=0$ ), which would allow the elimination of these differences. Thus, in each of the domains in the plane $\left(\kappa x, p_{x}\right)$, the particle trajectory is determined by the equation $I_{z}\left(\kappa x, p_{x}\right)=$ const, but values of $I_{z}$ are different in different domains. To plot such a trajectory in the $\left(\kappa x, p_{x}\right)$ plane, one needs to solve the equation $I_{z}\left(\kappa x, p_{x}\right)=$ const inside each domain and match these solutions at the uncertainty curve. It should be noted that in the $(\mathrm{t} 1)$ domain we actually have two non-identical equations $I_{z}\left(\kappa x, p_{x}\right)=$ const, corresponding to oscillations in the left and right potential wells, respectively (see schemes in Figs. 2 and 3 ).

The second effect for current sheets with $s \neq 0$ corresponds to the difference of the velocities $\Theta_{1, \mathrm{r}}$ (see Appendix A). For example, at the uncertainty curve one can find segments where $\Theta_{1}>0$ and $\Theta_{\mathrm{r}}<0$, or vice versa. The velocity of the area evolution for the single potential well $\Theta=-\left(\Theta_{1}+\Theta_{\mathrm{r}}\right)$ can be positive along such a segment. In this case, each trajectory coming to the uncertainty curve from the domain ( $\mathrm{t} 1)$ has two prolongations. The particle can go into the (t2) domain or stay in the (t1) domain but change the potential well. These two transitions are shown in Fig. 4 (middle panel, separatrix crossing C3). If the particle stays in the (t1) domain, after a certain time it approaches the uncertainty curve again or comes to the $(\mathrm{t} 2)$ domain without the uncertainty curve crossing. The latter variant is shown in Fig. 4 (middle panel, T2 transition), where the particle trajectory goes around the uncertainty curve and comes to the (t2) domain. Thus, at the uncertainty curve the adiabatic trajectory splits into two prolongations, and the particle may follow each of these prolongations with a certain probability (see description below). In the course of the particle's motion, this splitting at the uncertainty curve can occur several times. Due to this effect, any trajectory can be represented as a set of segments of trajectories in the various domains matched at several points at the uncertainty curve.

Hamiltonian (1) is invariant with respect to the transformation $z \rightarrow-z, p_{x} \rightarrow-p_{x}$. As a result the half-plane $\left(\kappa x, p_{x}\right), p_{x}>0$ is mirror symmetrical to the half-plane $p_{x}<0$. Therefore, all transitions from the (t1) domain to the (t2) domain with trajectory splitting are also mirror symmetrical relative to the line $p_{x}=0$. For example, should some particle approach the uncertainty curve inside the left well $(z<0)$ at $p_{x}=-p_{x}^{*}<0$, it can cross the uncertainty curve and get into the single well. Then this particle can approach the uncertainty curve inside the single well, cross the uncertainty curve at $p_{x}=p_{x}^{*}$, and get into the right well $(z>0)$.

Four examples of particle trajectories in the phase plane of slow variables are presented in Fig. 5 (here we describe trajectories crossing the uncertainty curve at some distance from its endpoints; see details in Sect. 7). General form of 

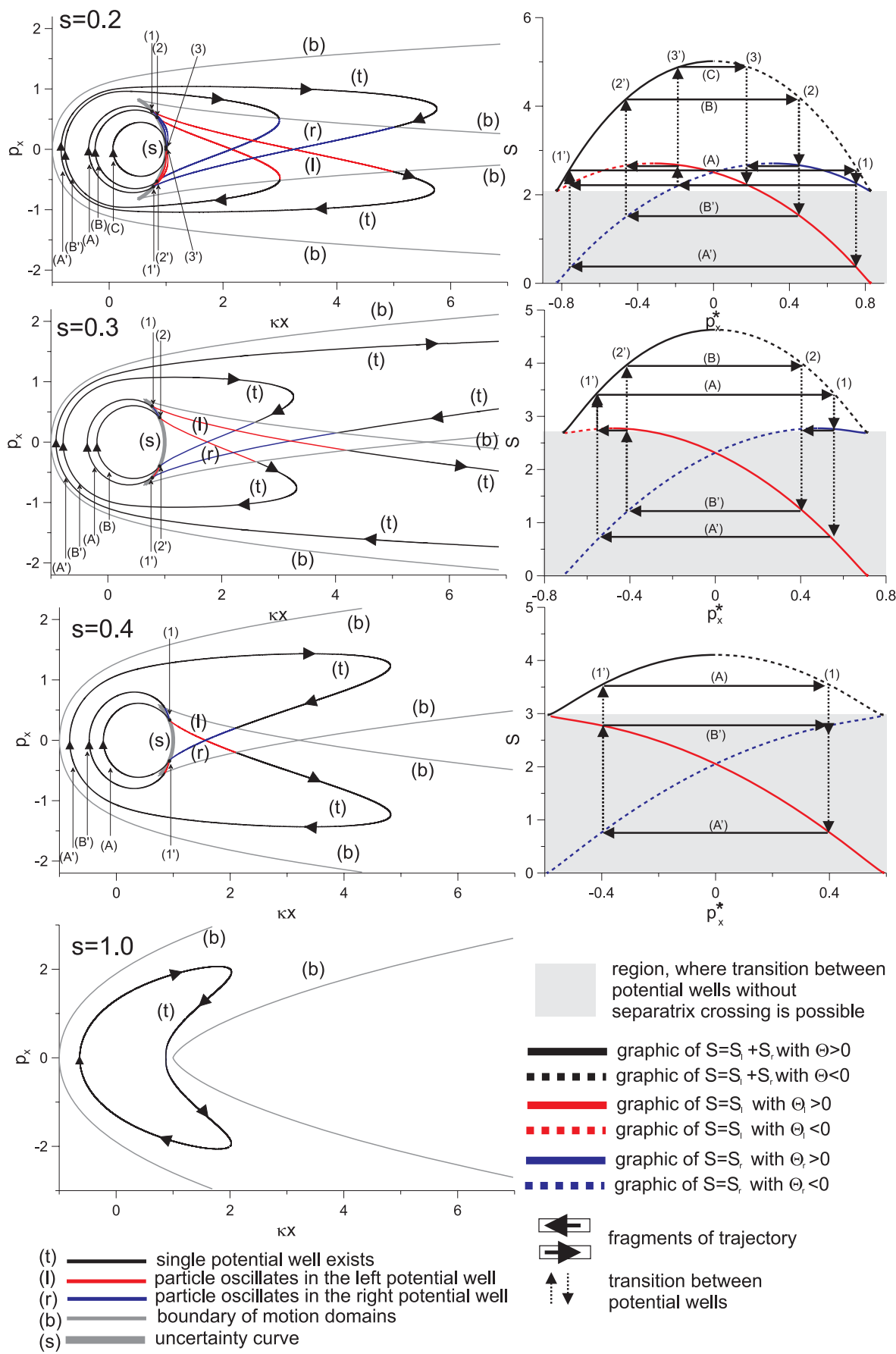

Fig. 5. Particle trajectories in the phase plane of the slow variables are shown for four values of the parameter $s$ (the left column). Corresponding profiles of $S_{1, \mathrm{r}}$ and $S$ are shown in the right column.

trajectories is defined by the number of the uncertainty curve crossings, because at these points trajectories split. The character of splitting and possible prolongations are determined by signs of $\Theta_{1, r}$ and $\Theta=-\left(\Theta_{1}+\Theta_{r}\right)$ at the corresponding point at the uncertainty curve. To describe these trajectories, we plot schemes with areas $S_{1, \mathrm{r}}$ and $S=S_{1}+S_{\mathrm{r}}$ as functions of the value $p_{x}^{*}$ along the uncertainty curve (see right panels in Fig. 5). We use dotted curves for the segments of graphs with negative values of $\Theta_{1, \mathrm{r}}, \Theta$ and solid curves for the segments with positive values of $\Theta_{1, \mathrm{r}}, \Theta$. Thus, dotted and solid curves correspond to the "ejecting" and "absorbing" segments of boundaries of domains, respectively.

Vertical dotted lines in the schemes (right panels) correspond to the particle transitions between the potential wells 
(one line corresponds to one point at the uncertainty curve, where the trajectory crosses it). Horizontal solid lines correspond to segments of particle trajectories. Particles in this scheme move along the horizontal straight lines up to the crossing of $S_{1, \mathrm{r}}$ profiles. Then particles should change the potential well (i.e. particles reach the uncertainty curve).

There is a new effect for the system with $s \neq 0$. Particles can switch between the right and left potential wells without the uncertainty curve crossing (i.e. without the separatrix crossing). Corresponding lines in the schemes in the right panels of Fig. 5 are inside the grey region. The particle can transit between two wells according to the following scenario: initially, the particle oscillates in the left well. Then the right well disappears and the left well with the oscillating particle shifts toward the right well position. Then new left well appears. The result is the transit of the particle from the left to the right well without separatrix crossing, i.e. at all times the particle oscillates in the same well, which changes its position from left to right. To clarify this description, we present a scheme with successive profiles of $U(z)$ and corresponding particle position for two transitions from the left well to the right well with and without separatrix crossings (see Fig. 6).

Because $S_{1} \neq S_{\mathrm{r}}$, particles change the value of $I_{z}$ due to the transition between potential wells at the separatrix. As a result, we have a non-zero value for the geometrical jump $\Delta I_{z}^{\text {geom }}$.

The splitting of the possible routes of particle motion at the uncertainty curve means that the corresponding trajectory of the full system should choose one of two possible prolongations. This choice depends on values of the fast variables at the moment of the separatrix crossing in the plane $\left(z, p_{z}\right)$. Because variables $\left(z, p_{z}\right)$ change (approximately) periodically with periods smaller than time scale of $S_{1, \mathrm{r}}$ evolution, these values may be considered as random. In this case each choice of the trajectory prolongation at the uncertainty curve has some probability. These probabilities depend on the crossing position on the uncertainty curve. In particular, such probabilities can be considered as functions of the coordinate $p_{x}=p_{x}^{*}$ along the uncertainty curve (see Appendix B).

One can find three types of trajectories in Fig. 5.The first type corresponds to trajectories presented in panels with $s=0.2$ and $s=0.3$. Trajectories of this type cross the uncertainty curve several times and have two transitions through the $(\mathrm{t} 2)$ domain with the single potential well without uncertainty curve crossings. The second type of trajectories is shown for the system with $s=0.4$. These trajectories cross the uncertainty curve only twice (on entering the (t2) domain with the single well and at the exit from this domain). Particles moving along such a trajectory always come to the uncertainty curve at points where $\Theta_{\mathrm{r}}<0$. Thus, transit into the right well is possible only without separatrix crossing (see the scheme in Fig. 6, right panels). This type of trajectory exists for $s$ larger than a certain threshold. The third type of

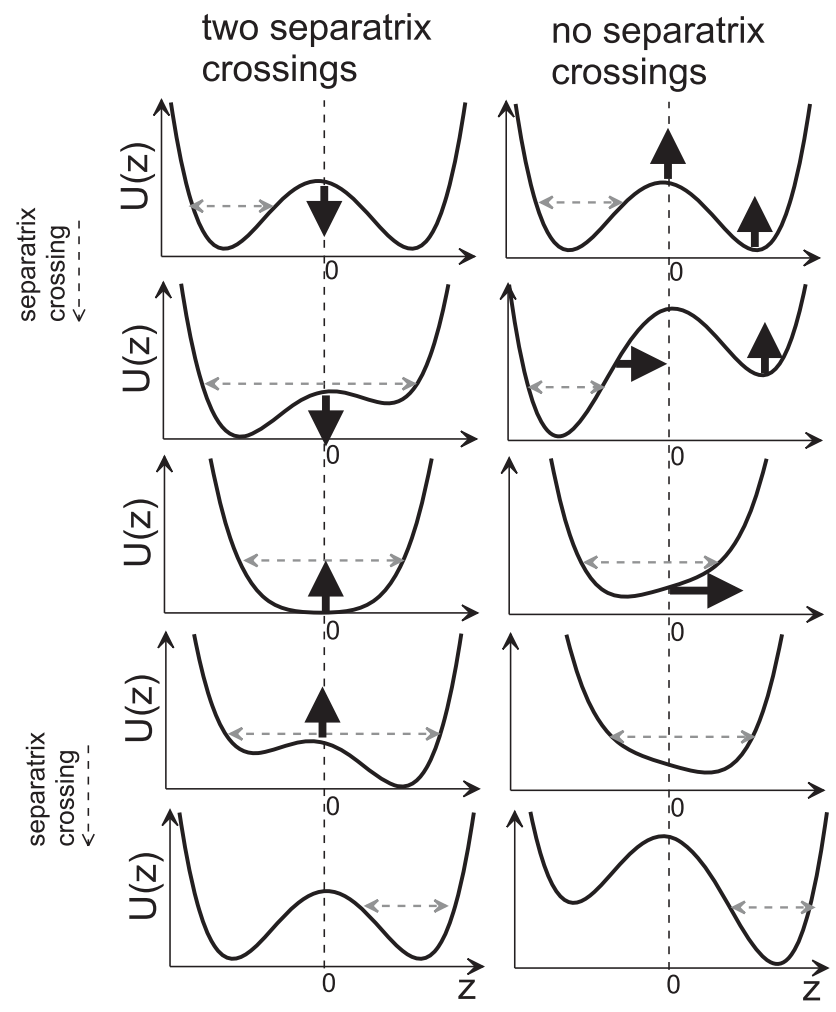

Fig. 6. Left column shows successive profiles of $U(z)$ and particle positions (from top to bottom) for transition from left well to right well with two separatrix crossings. Right column shows the same transition, but without separatrix crossings. Grey arrows correspond to particle position $U=1 / 2$ and black arrows show direction of $U(z)$ deformation

trajectory is presented for $s=1$. In this case the uncertainty curve (and the separatrix) is absent, and the trajectory is a single closed curve with the same $I_{z}=$ const along it. The transition from regime $s<1$ to $s \geq 1$ corresponds to disappearance of the uncertainty curve.

The existence of various types of trajectories is determined by the value of the parameter $s$. If $s=0$ we have the system with two domains in the plane $\left(\kappa x, p_{x}\right)$ and $S_{1}=S_{\mathrm{r}}$. In this case all trajectories are closed curves with $I_{z}=$ const. In systems with $s \neq 0$, as long as $s$ is smaller than a certain value $s_{\text {bif }}$, only trajectories of the first type exist. All trajectories have the same number of uncertainty curve crossing points for a fixed $s$ (see Appendix C). Examples of these trajectories are shown in Fig. 5 for $s=0.2$ and $s=0.3$. Value $s_{\text {bif }} \approx 0.25$ corresponds to the system where the minimum value of $S=S_{1}+S_{\mathrm{r}}$ is equal to the value of $S_{1, \mathrm{r}}$ at $p_{x}^{*}=0$. In systems with $s_{\mathrm{bif}}<s<1$ we can observe a new type of trajectory (shown in Fig. 5 for $s=0.4$ ). Trajectories of the first type disappear for $s$ larger than a certain value $\bar{s}$. This value $\bar{s} \approx 0.35$ corresponds to the system where $\Theta_{\mathrm{r}}$ is negative everywhere along the uncertainty curve. Therefore, in the system with $s_{\mathrm{bif}}<s<\bar{s}$ trajectories of both types (first 
and second) can be found. In the system with $1>s>\bar{s}$ only trajectories of the second type exist. In the system with $s \geq 1$ the separatrix disappears and we have only trajectories of the third type (shown in Fig. 5 for $s=1.0$ ). Evaluation of bifurcation values of the parameter $s$ is described in Appendix A. We summarize all characteristics of systems with various $s$ in Table 1.

\section{Trajectories in 3-D}

We compare trajectories obtained in the adiabatic approximation with numerical solutions of the equations of motion for Hamiltonian (1). We choose a small value of the parameter $\kappa$ to make dynamical jumps negligibly small. To illustrate the effect of the trajectory splitting at the uncertainty curve, we calculate two trajectories starting from the same point in the $\left(\kappa x, p_{x}\right)$ plane with the same energy, but with different values of the fast coordinates (trajectories (1) and (2) in each panel of Fig. 7). Examples of such trajectories are shown for systems with $s=0.3$ and $s=0.4$. We also show the splitting in separated panels.

One can observe that the particle chooses one of two prolongations of the trajectory at the uncertainty curve. Roughly speaking, both trajectories can be initiated at the same point of the uncertainty curve (splitting points shown in Fig. 7), but their further prolongation would be different. Choice of prolongation depends on values of the fast variables. Shapes of the trajectories are similar to ones obtained in the adiabatic approximation in the previous section, because the influence of dynamic jumps was negligible.

\section{Discussion}

The Hamiltonian system (1) has four variables $\left(z, p_{z}, \kappa x, p_{x}\right)$. However, due to conservation of energy $H$, the dimension of the system can be reduced to 3-D. Therefore, any curve in the phase plane $\left(\kappa x, p_{x}\right)$ of slow variables corresponds to some surface in this 3-D space. Without loss of generality we consider the energy level $H=1 / 2$ (we have the equation $H\left(z, p_{z}, \kappa x, p_{x}\right)=\mathrm{const}$ and use normalization to obtain $H=1 / 2$ ). The obtained 3-D volume $\Lambda$ is filled by trajectories of the fast motion. These are trajectories of the system with Hamiltonian (1) at various frozen variables $\left(\kappa x, p_{x}\right)$. Figure 3 is a projection of $\Lambda$ onto the plane $\left(\kappa x, p_{x}\right)$. Thus, each trajectory of the fast system is projected to a point in this plane. However, to each point in the domain (t1) in Fig. 3, two trajectories of the fast system are mapped. Those are trajectories of oscillations in the left and right potential wells with the same energy $H=1 / 2$. A convenient way to represent the dynamics is to consider two copies, (t11) and (t1r), of the domain (t1). In this case those trajectories of oscillations in the left and right potential wells are projected to (t11) and (t1r), respectively. Then we glue (t1l) with (t2l), and (t1r) with (t2r). We also glue (t1l)
Table 1. System characteristics for various $s$.

\begin{tabular}{ll}
\hline$s$ range & Description \\
\hline$s=0$ & $\begin{array}{l}\text { all trajectories are closed curves with two } \\
\text { crossings of the uncertainty curve }\end{array}$ \\
$0<s<s_{\text {bif }}$ & $\begin{array}{l}\text { only trajectories of the first type exist } \\
\text { (see example in Fig. 5, } s=0.2 \text { ) }\end{array}$ \\
$s_{\text {bif }}<s<\bar{s}$ & $\begin{array}{l}\text { trajectories of the first and second types exist } \\
\text { (see example in Fig. 5, } s=0.4 \text { ) }\end{array}$ \\
$\bar{s}<s<1$ & $\begin{array}{l}\text { only trajectories of the second type exist } \\
s \geq 1\end{array}$ \\
& $\begin{array}{l}\text { only trajectories of the third type exist } \\
\text { (see example in Fig. 5, } s=1.0 \text { ) }\end{array}$ \\
\hline
\end{tabular}

with ( $\mathrm{t} 1 \mathrm{r}$ ) along their edges corresponding to the uncertainty curve, which is the projection of separatrices. The obtained irregular 2-D surface is a phase space of the slow system. One can show that this surface relates to the classical object of catastrophe theory - the swallowtail surface (see Arnold, 1992).

In this paper we found the critical value of the parameter $s$ where the uncertainty curve (and the separatrix) vanishes. We show that the uncertainty curve does not exist in systems with $s \geq 1$ (see Appendix A). In dimensional variables this criterion can be written as $2 H<m\left(\omega_{0} L\right)^{2}\left(B_{y} / B_{0}\right)^{4}$ or $B_{y}>B_{0} \sqrt{\rho_{0} / L}$, where $\omega_{0}=q B_{0} / m c$. The same criterion of particle magnetization by $B_{y}$ component was obtained earlier by Galeev and Zelenyi (1978) and widely used in the theory of current sheet instability (see e.g. Zelenyi and Taktakishvili, 1988, and references therein). For a given value of $B_{y}$, particles with energy $2 H<m\left(\omega_{0} L\right)^{2}\left(B_{y} / B_{0}\right)^{4}$ are not scattered in the current sheet ( $\Delta I_{z}^{\text {geom }}$ and $\Delta I_{z}^{\text {dyn }}$ are absent). Their motion can be described as adiabatic with $I_{z}=$ const. One can easily show that for systems with $s \gg 1$ the guiding center theory is applicable. In this case the invariant $I_{z}$ is proportional to the magnetic moment.

The system under consideration contains a class of regular trajectories in the domain ( $\mathrm{t} 2)$ with the single potential well called "ring" orbits (see details in Büchner and Zelenyi, 1986; Chen and Palmadesso, 1986; Büchner and Zelenyi, 1989; Chen, 1992). Particles on these trajectories cross the neutral plane $z=0$ twice at each period of the fast oscillations. These trajectories never cross the uncertainty curve. As a result for these trajectories $\Delta I_{z}^{\text {dyn }}$ and $\Delta I_{z}^{\text {geom }}$ are absent. Phase volume filled by regular trajectories depends on the length of the uncertainty curve $\ell$ in the $\left(\kappa x, p_{x}\right)$ plane. For the system with $s=0$, regular trajectories occupy less than $20 \%$ of the phase volume (this volume can be larger for a bifurcated current sheet, see Artemyev et al., 2011; Vasiliev et al., 2012). However, as we show in Appendix A, length $\ell$ decreases with the increase of the parameter $s$. For systems with $s \geq 1$ the separatrix vanishes. Thus, this class of 

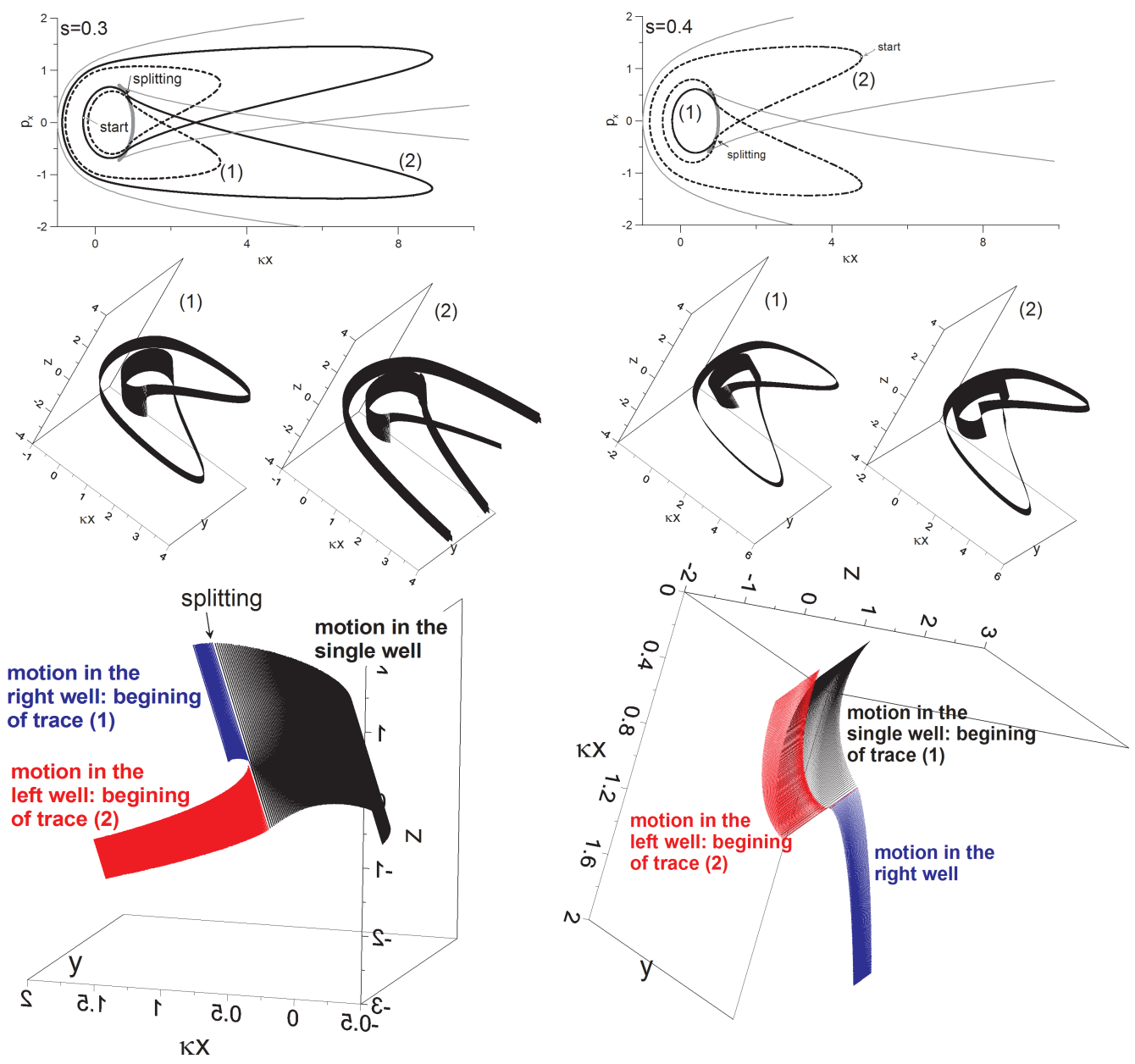

Fig. 7. Trajectories in 3-D obtained by numerical integration of the Hamiltonian equations are shown for $s=0.3$ and $s=0.4$. Projections of these trajectories onto $\left(\kappa x, p_{x}\right)$ plane are also shown. In 3-D we demonstrate only segments of each trajectory corresponding to the top panels with projections. Bottom colour panels show moments of trajectory splitting for both values of $s$.

trajectories (those crossing the neutral plane $z=0$ twice on each period of their fast oscillations) does not exist. In systems with $s \geq 1$ all trajectories are regular (i.e. $\Delta I_{z}^{\text {dyn }}$ and $\Delta I_{z}^{\mathrm{geom}}$ are absent), but all particles leave the vicinity of the neutral plane $z=0$ moving along field lines (an example of such a trajectory is shown in Fig. 1d).

In Appendix B we determine the probabilities of arrival into different domains after crossing the uncertainty curve. These probabilities depend on the coordinate $p_{x}^{*}$ along the uncertainty curve. Using these probabilities one can calculate the probability for any trajectory consisting of several segments matched at the uncertainty curve. One needs to multiply the corresponding probabilities of transitions between domains in the $\left(\kappa x, p_{x}\right)$ plane at the uncertainty curve for such a multisegmental trajectory. This procedure can be used to obtain the reflection and transition coefficients (portions of particles which will return to the initial half-space relative to $z=0$ or pass through the current sheet and appear at the opposite half-space). We left this topic for further publication.
In this paper we pay more attention to the geometrical jumps. We assume that $\Delta I_{z}^{\text {geom }}$ is more important in comparison with the dynamical jumps $\Delta I_{z}^{\mathrm{dyn}}$. However, for the system with a small value of the parameter $s$, the influence of $\Delta I_{z}^{\mathrm{dyn}}$ can be comparable with one of $\Delta I_{z}^{\text {geom }}$. To estimate the critical value of $s$, we obtain the approximation of $\Delta I_{z}^{\text {geom }}$ for small $s$ (see Appendix D). One can conclude that in the system with $s>(2 / \pi) \kappa \ln 2$, the shape of particle trajectories is defined by the geometrical jumps. Dynamical jumps lead to a slow diffusion across adiabatic trajectories (i.e. to a slow evolution of real particle trajectories).

Particles cross the uncertainty curve at each period of slow variables oscillations (the duration of this period is proportional to $\kappa^{-1}$ ). Thus, geometrical jumps modify the particle trajectory (and the value of quasi-adiabatic invariant) at each period. Simultaneously particles slightly change their trajectories due to dynamical jumps $\sim \kappa \ln \kappa$ (Neishtadt, 1986; Cary et al., 1986). Each separatrix crossing at $p_{x}=p_{x}^{*}$ is accompanied later by the second separatrix crossing at 
$p_{x}=-p_{x}^{*}$ at the same adiabatic trajectory (crossings occur both at enter to and at exit from (t2) domain). The sum of these two consecutive dynamical jumps can be considered as a random value with a zero mean. Thus the effect of the dynamical jumps will be considerable only after $\sim \kappa^{-2} \ln ^{-2} \kappa$ separatrix crossings. This process takes time $\sim \kappa^{-3} \ln ^{-2} \kappa$, while even a single geometric jump produces a considerable change of the quasi-adiabatic invariant $I_{z}$ in general. However, the role of geometrical and dynamical jumps in the considered system is completely different. Geometrical jumps lead to the formation of adiabatic trajectories in the plane of slow variables. These trajectories are made of segments glued together at the uncertainty curve. Phase point moves along these trajectories switching randomly between segments. Dynamical jumps lead to a diffusion across adiabatic trajectories and stochastization of particle motion.

Charged particle dynamics in the magnetotail current sheet with $B_{y} \neq 0$ was studied before by means of numerical integration of trajectories (Karimabadi et al., 1990; Büchner and Zelenyi, 1991; Zhu and Parks, 1993; Kaufmann et al., 1994; Baek et al., 1995; Holland et al., 1996; Chapman and Rowlands, 1998; Delcourt et al., 2000; Ynnerman et al., 2000). We can compare predictions of our analytical theory with results obtained in these investigations. Five main effects of $B_{y}$ were found by numerical integration of particle trajectories: (1) Karimabadi et al. (1990) and Büchner and Zelenyi (1991) showed that particles are not scattered in the current sheet for large enough $B_{y}$, i.e. jumps of the quasi-adiabatic invariant are absent. Büchner and Zelenyi (1991) determined the critical value of $B_{y}$ for this transition to the regime of regular motion as $B_{y}>B_{0} \sqrt{L / \rho_{0}}$. This value coincides with our estimates of $B_{y}$ corresponding to vanishing of the separatrix. Therefore, we can explain absence of particle scattering by the absence of the separatrix in the phase plane $\left(z, p_{z}\right)$ at large enough $B_{y}$. Moreover, Karimabadi et al. (1990) and Holland et al. (1996) pointed out that scattering of particles is not influenced by $B_{y}$ while $B_{y}<B_{z}$. Our theory gives the more accurate criterion $B_{y}<B_{z}(2 / \pi) \ln 2$ (i.e. $B_{y}<0.44 B_{z}$ ); (2) in this paper we consider only adiabatic theory without detailed analysis of destruction of the quasi-adiabatic invariant. Thus, the effect of intensification of particle scattering for $B_{y} \sim B_{z}$ is beyond the scope of our paper. This effect was found by Karimabadi et al. (1990); Holland et al. (1996); Chapman and Rowlands (1998); (3) asymmetry of particle trajectories with respect to the neutral plane $z=0$ has been mentioned by many authors. Zhu and Parks (1993) numerically integrated particle trajectories in the current sheet with $B_{y} \sim B_{z} \ll B_{0}$. They showed that particles escape from the current sheet mainly to one hemisphere $(z>0$ or $z<0$ depending on the sign of $B_{y}$ ). Moreover, Baek et al. (1995) demonstrated that at $B_{y}$ larger than a certain value, particles escape from the current sheet only to one hemisphere. Our theory describes this effect of asymmetry. Direction of particle escape from the current sheet is determined by probabilities of particle capture into left and right potential wells. Increase of $B_{y}$ results in decrease of one of these probabilities (see Appendix B). Thus, particle captures are asymmetrical at $B_{y} \neq 0$. Moreover, if $B_{y}>0.35 B_{0} \sqrt{L / \rho_{0}}$, particles can be captured only in one potential well. Therefore, all particles escape from the current sheet to one hemisphere. Delcourt et al. (2000) found an interesting feature of asymmetry of particle scattering in the current sheet with $B_{y} \neq 0$. They showed that particles are not scattered for a certain range of initial pitch angles. We also can describe this asymmetry of scattering. Length of the uncertainty curve in the $\left(\kappa x, p_{x}\right)$ plane decreases with increase of $B_{y}$ (see Appendix A). As a result some particle trajectories can cross the current sheet without crossing the uncertainty curve (thus, without jumps of the quasi-adiabatic invariant, see Fig. 5). Particles moving along these trajectories are not scattered at one current sheet crossing; (4) Kaufmann et al. (1994) and Holland et al. (1996) described an effect of destruction of particle resonant interaction with the current sheet in case of $B_{y} \neq 0$. Resonant interaction corresponds to particle motion without dynamical jumps of the quasi-adiabatic invariant (two successive jumps $\Delta I_{z}^{\text {dyn }}$ compensate each other and $I_{z}$ is conserved; see details in Büchner and Zelenyi, 1989). However, in this paper we restrict our investigation to adiabatic theory. Thus, this effect is beyond the scope of our investigation; (5) Chapman and Rowlands (1998) and Ynnerman et al. (2000) considered the effect of $B_{y}$ in regular particle trajectories, which never cross the uncertainty curve (i.e. never cross the separatrix). Our theory is able to describe the decrease in number of such trajectories with the increase of $B_{y}$ (this effect was found by Chapman and Rowlands, 1998). This decrease corresponds to decrease of length of the uncertainty curve. However, we did not develop a detailed theory for this class of trajectories in the case of $B_{y} \neq 0$. Thus, we do not consider all peculiarities of these trajectories reported by Ynnerman et al. (2000).

We study particle trajectories that cross the uncertainty curve not very close to its endpoints (all crossings correspond to $\left|p_{x}^{*}\right|$ far from $\pm \max p_{x}^{*}$ ). Thus, particle dynamics in the vicinity of the endpoints of the uncertainty curve is beyond the scope of this paper. In the close vicinity of the endpoints of the uncertainty curve, velocities $\Theta_{1, \mathrm{r}}$ change the sign (see Fig. A3 in Appendix A). Related dependence of $S_{1, \mathrm{r}}$ on $p_{x}^{*}$ is not monotonous. Particles crossing the uncertainty curve in this region have specific trajectories. However, due to smallness of this region in the phase plane (the length of the corresponding segment of the uncertainty curve is smaller than $1 \%$ of its entire length), the role of such trajectories seems to be unimportant.

The effect of multiple geometrical jumps in the systems with periodical separatrix crossings was discussed earlier by Neishtadt (1986). In this paper we consider a particular physical system with multiple geometrical jumps. The invariance of Hamiltonian (1) to transformation $p_{x} \rightarrow-p_{x}, z \rightarrow-z$ results in an additional symmetry of the geometrical jumps. 
Finally, all (split) trajectories have a finite number of uncertainty curve crossings (i.e. trajectories are closed). In a general situation consecutive geometrical jumps should lead to an infinite number of uncertainty curve crossings and correspondingly to very fast chaotization (Neishtadt and Treschev, 2011).

\section{Conclusions}

In this paper we study ion dynamics in current sheets with $B_{y}$ component of the magnetic field, often called current sheets with sheared magnetic field. We describe particle trajectories in adiabatic approximation and demonstrate that a principal role is played by geometrical jumps of the quasi-adiabatic invariant. We found four regimes of the system depending on the parameter $s=\left(B_{y} / B_{0}\right) \sqrt{L / \rho_{0}}$ (see, also, Table 1):

1. $s \in\left(0, s_{\text {bif }}\right)$ : only one type of trajectory exists; number of uncertainty curve crossings depends on $s$ value (see Fig. C1).

2. $s \in\left(s_{\mathrm{bif}}, \bar{s}\right)$ : two types of trajectories exist; trajectories of the second type cross uncertainty curve only twice (once for $p_{x}^{*}>0$ and once for $p_{x}^{*}<0$ ).

3. $s \in(\bar{s}, 1.0)$ : trajectories of the first type do not exist, and all trajectories correspond to the second type.

4. $s \geq 1$ : the separatrix vanishes and the geometrical (as well as dynamical) jumps of the quasi-adiabatic invariant disappear; for $s \gg 1$ one can obtain transition to the guiding-centre theory.

Analytical estimates give $s_{\text {bif }} \approx 0.25$ and $\bar{s} \approx 0.35$, or $\left(B_{y}\right)_{\text {bif }} \approx 0.25 B_{0} \sqrt{L / \rho_{0}}$ and $\bar{B}_{y} \approx 0.35 B_{0} \sqrt{L / \rho_{0}}$. Comparison of the dynamical and the geometrical jumps shows that at $s<(2 / \pi) \kappa \ln 2$ geometrical jumps are smaller than the dynamical jumps. In this case the role of geometrical jumps can be neglected. Thus, particle dynamics is similar to one for the system with $s=0$. We conclude that the concept of geometrical jumps is very practical and allow the understanding of the differences between system with $s=0$ and $s \neq 0$.

\section{Appendix A}

\section{The separatrix}

In Fig. 2 one can see the saddle point with coordinates $z=z_{\mathrm{c}}$, $p_{z}=0$ at the separatrix. At this point the potential energy $U$ has a local maximum value; thus, $\partial U / \partial z=0$. We use this condition to express slow coordinates along the uncertainty curve in the plane $\left(\kappa x, p_{x}\right)$ as functions of $z_{\mathrm{c}}$. The equation for the uncertainty curve can be written as $1=p_{z}^{2}+\left(p_{x}^{*}-\right.$ $s z)^{2}+\left(\kappa x^{*}-\frac{1}{2} z^{2}\right)^{2}$, where $z$ and $p_{z}$ are coordinates of any
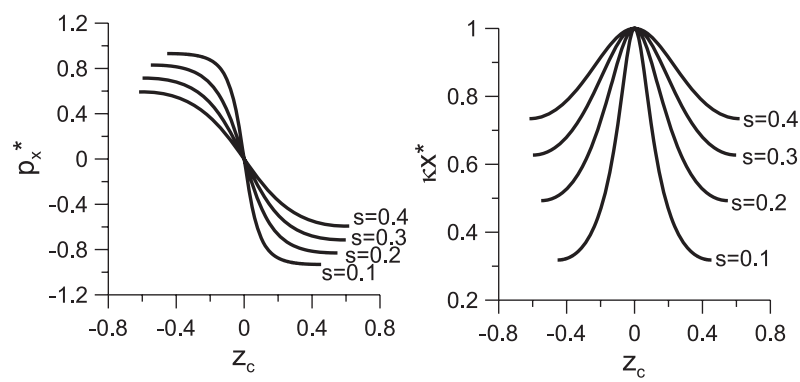

Fig. A1. Slow coordinates $p_{x}^{*}, \kappa x^{*}$ along the uncertainty curve are shown as functions of $z_{\mathrm{c}}$ for various $s$.

point on the separatrix. Here we use $z=z_{\mathrm{c}}$ and $p_{z}=0$ and obtain:

$$
\begin{aligned}
& p_{x}^{*}=s z_{\mathrm{c}}-\frac{z_{\mathrm{c}}}{s} A_{z_{\mathrm{c}}} \\
& \kappa x^{*}=\frac{1}{2} z_{\mathrm{c}}^{2}+A_{z_{\mathrm{c}}} \\
& A_{z_{\mathrm{c}}}=\left(1+\frac{z_{\mathrm{c}}^{2}}{s^{2}}\right)^{-1 / 2} .
\end{aligned}
$$

Note that in the system with $B_{y}=0$ we have $s=0, z_{\mathrm{c}}=0$. In this case the uncertainty curve in the phase plane of the slow variables is the half-circle $\left(\kappa x^{*}\right)^{2}+\left(p_{x}^{*}\right)^{2}=1, \kappa x^{*}>0$. In the system under consideration, the position of the saddle point $z_{\mathrm{c}}$ depends on coordinates along the uncertainty curve $\left(\kappa x^{*}, p_{x}^{*}\right)$, see Fig. A1.

With increase of $s$, the range of $p_{x}^{*}$ variation shrinks. The length of the uncertainty curve in the plane $\left(\kappa x, p_{x}\right)$ can be calculated as

$$
\begin{aligned}
\ell(s) & =\int_{-z_{\mathrm{cmax}}}^{z_{\mathrm{cmax}}} \sqrt{1+\left(\partial p_{x}^{*} / \partial \kappa x^{*}\right)^{2}}\left(\partial \kappa x^{*} / \partial z_{\mathrm{c}}\right) \mathrm{d} z_{\mathrm{c}} \\
= & 2 \arctan \left(z_{\mathrm{cmax}} / s\right)-z_{\mathrm{cmax}}^{2} \sqrt{1+\left(s / z_{\mathrm{cmax}}\right)^{2}} \\
= & 2 \arctan \left(\sqrt{s^{-4 / 3}-1}\right)-s^{1 / 3} \sqrt{s^{2 / 3}-s^{2}} .
\end{aligned}
$$

Here we use the following expression for the maximum value of $z_{\mathrm{c}}: z_{\mathrm{cmax}}=\sqrt{s^{2 / 3}-s^{2}}$ (this expression is derived below). One can easily obtain that $\ell(0)=\pi$. For $s=1$ we have $\ell(1)=0$. Thus, the uncertainty curve (as well as the separatrices) vanishes at $s=1$ (see Fig. A2).

In this Appendix we derive expressions for areas $S_{1, \mathrm{r}}$ surrounded by two separatrix loops and velocities $\Theta_{1, \mathrm{r}}$ of their evolution (all these values depend on a slow coordinate along the uncertainty curve). For $S_{1, \mathrm{r}}$ one can write

$$
S_{1, \mathrm{r}}=2 \int_{z_{1, \mathrm{~m}}}^{z_{1, \mathrm{r}}^{\max }} p_{z} \mathrm{~d} z=2 \int_{z_{\mathrm{min}}}^{z_{1, \mathrm{r}}^{\max }} \sqrt{2 H-2 U\left(\kappa x^{*}, p_{x}^{*}, z\right)} \mathrm{d} z,
$$

where the limits of integration are $z_{1, \mathrm{r}}^{\max }=z_{\mathrm{c}}, z_{+}, z_{1, \mathrm{r}}^{\min }=$ $z_{-}, z_{\mathrm{c}}$. Values of $z_{ \pm}$are defined by the equation $H=U\left(\kappa x^{*}, p_{x}^{*}, z_{ \pm}\right)$. We integrate along the separatrix and 


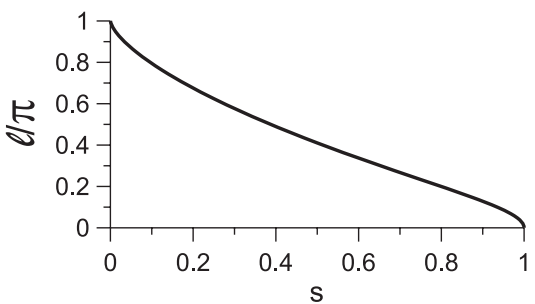

Fig. A2. The length of the uncertainty curve is shown as function of $s$.

can take $H=U_{\mathrm{c}}=U\left(\kappa x^{*}, p_{x}^{*}, z_{\mathrm{c}}\right)$ :

$2 U_{\mathrm{c}}-2 U\left(\kappa x, p_{x}, z\right)=\left(z-z_{\mathrm{c}}\right)^{2}\left[A_{z_{\mathrm{c}}}-s^{2}-\frac{1}{4}\left(z_{\mathrm{c}}+z\right)^{2}\right]$.

After integration we have

$$
\begin{aligned}
S_{1, \mathrm{r}} & =-A_{s} \pm \frac{\pi}{2} z_{\mathrm{c}} \tilde{\zeta}^{2} \\
A_{s} & =-z_{\mathrm{c}} \tilde{\zeta}^{2} \arctan \left(\frac{2 z_{\mathrm{c}}}{\sqrt{\tilde{\zeta}^{2}-4 z_{\mathrm{c}}^{2}}}\right) \\
& -2 z_{\mathrm{c}}^{2} \sqrt{\tilde{\zeta}^{2}-4 z_{\mathrm{c}}^{2}}-\frac{1}{3}\left(\tilde{\zeta}^{2}-4 z_{\mathrm{c}}^{2}\right)^{3 / 2},
\end{aligned}
$$

where $\tilde{\zeta}=2 \sqrt{A_{z_{\mathrm{c}}}-s^{2}}$. From Eq. (A3) one can define the range of $z_{\mathrm{c}}$ variation: $z_{\mathrm{c}} \in\left[-\sqrt{s^{2 / 3}-s^{2}}, \sqrt{s^{2 / 3}-s^{2}}\right]$.

Expressions for $\Theta_{1, \mathrm{r}}=\mathrm{d} S_{1, \mathrm{r}} / \mathrm{d} \kappa t$ can be found with the use of the following formula (see details in Neishtadt, 1986; Arnold et al., 2006):

$\Theta_{1, \mathrm{r}}=\oint\left(\frac{\partial U}{\partial p_{x}^{*}} \frac{\partial U_{\mathrm{c}}}{\partial \kappa x^{*}}-\frac{\partial U}{\partial \kappa x^{*}} \frac{\partial U_{\mathrm{c}}}{\partial p_{x}^{*}}\right) \mathrm{d} t$

where we integrate along the separatrix loop (left or right). We can write

$\frac{\partial U}{\partial p_{x}^{*}} \frac{\partial U_{\mathrm{c}}}{\partial \kappa x^{*}}-\frac{\partial U}{\partial \kappa x^{*}} \frac{\partial U_{\mathrm{c}}}{\partial p_{x}^{*}}=2 A_{z_{\mathrm{c}}}\left(z-z_{\mathrm{c}}\right)\left(2 s+\frac{z_{\mathrm{c}}}{s}\left(z+z_{\mathrm{c}}\right)\right)$.

We replace the integration over time by the integration over the fast variable, $\mathrm{d} t=\mathrm{d} z / p_{z}$, and get

$$
\begin{aligned}
& \Theta_{1, \mathrm{r}}=4 A_{z_{\mathrm{c}}}\left(A_{\Theta} \pm \frac{\pi}{2} s\right) \\
& A_{\Theta}=s \arctan \left(\frac{2 z_{\mathrm{c}}}{\sqrt{\tilde{\zeta}^{2}-4 z_{\mathrm{c}}^{2}}}\right)-\frac{1}{2} \frac{z_{\mathrm{c}}}{s} \sqrt{\tilde{\zeta}^{2}-4 z_{\mathrm{c}}^{2}} .
\end{aligned}
$$

The area surrounded by two loops is $S=S_{1}+S_{\mathrm{r}}$. We denote $\Theta=-\left(\Theta_{1}+\Theta_{\mathrm{r}}\right)$. We plot these values as functions of $p_{x}^{*}$ in Fig. A3.

From Fig. A3 one can see that there is a specific value $\bar{s}$ of the parameter $s$. For $s>\bar{s}$ we have $\Theta_{\mathrm{r}}<0$ (and $\Theta_{1}>0$ ) everywhere along the uncertainty curve. To determine $\bar{s}$ we note that for $s=\bar{s}$ there is some point $p_{x}^{*}=\bar{p}_{x}^{*}$, where $\Theta_{\mathrm{r}}=$ 0 and $\partial \Theta_{\mathrm{r}} / \partial p_{x}^{*}=0$ (see Fig. A3). We can replace $\partial / \partial p_{x}^{*}$ by $\partial / \partial z_{\mathrm{c}}$ in view of Eq. (A1). In this case we obtain the
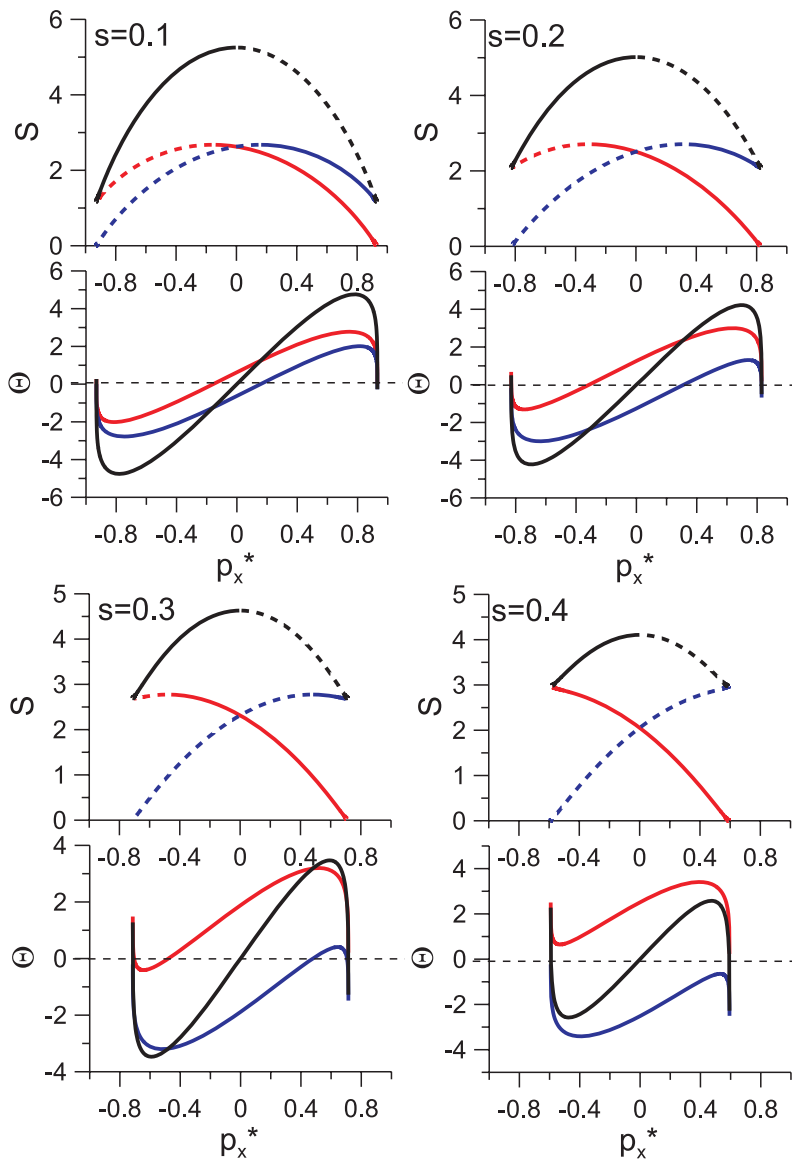

Fig. A3. Areas $S_{1}$ (red), $S_{\mathrm{r}}$ (blue), $S$ (black) and velocities $\Theta_{i}$ are shown as functions of the parameter $p_{x}^{*}$ along the uncertainty curve for various $s$. Dotted segments of curves $S_{i}$ correspond to regions with $\Theta_{i}<0$.

following system of equations:

$A_{\Theta}\left(\bar{z}_{\mathrm{c}}, \bar{s}\right)=\frac{\pi}{2} \bar{s}$

$\partial A_{\Theta} /\left.\partial z_{\mathfrak{c}}\right|_{\bar{z}_{\mathfrak{c}}, \bar{s}}=0$

$\bar{p}_{x}=\bar{s} \bar{z}_{\mathrm{c}}-\frac{\bar{z}_{\mathrm{c}}}{\bar{s}} A_{z_{\mathrm{c}}}$.

The solution of this system is $\bar{s} \approx 0.35, \bar{p}_{x}^{*} \approx 0.6$ and $\bar{z}_{\mathrm{c}} \approx$ -0.35 .

Another important value of $s$ can be found from the equation $\ell(s)=0$ (i.e. the separatrix vanishes). Equation (A2) gives $s=1$ for $\ell(s)=0$. For a system with $s \geq 1$, a separatrix does not exist. As a result $\Delta I_{z}^{\text {geom }}$ and $\Delta I_{z}^{\text {dyn }}$ are absent.

The last value of the parameter $s$ where bifurcation of the system occurs is $s=s_{\text {bif }}$. In the system with $s<s_{\text {bif }}$ only one type of particle trajectory exists and in the system with $s>s_{\text {bif }}$ we have two types of particle trajectories. The value of $s_{\text {bif }}$ can be defined as follows: when $s=s_{\text {bif }}$ the minimum value of $S=S_{1}+S_{\mathrm{r}}$ is equal to the values of $S_{1, \mathrm{r}}$ at the point $p_{x}^{*}=0$. The minimum value of $S=S_{1}+S_{\mathrm{r}}$ is $4 \pi\left(s^{2 / 3}-s^{2}\right)^{3 / 2}$. Values of $S_{1, \mathrm{r}}$ at $p_{x}^{*}=0$ are equal to 

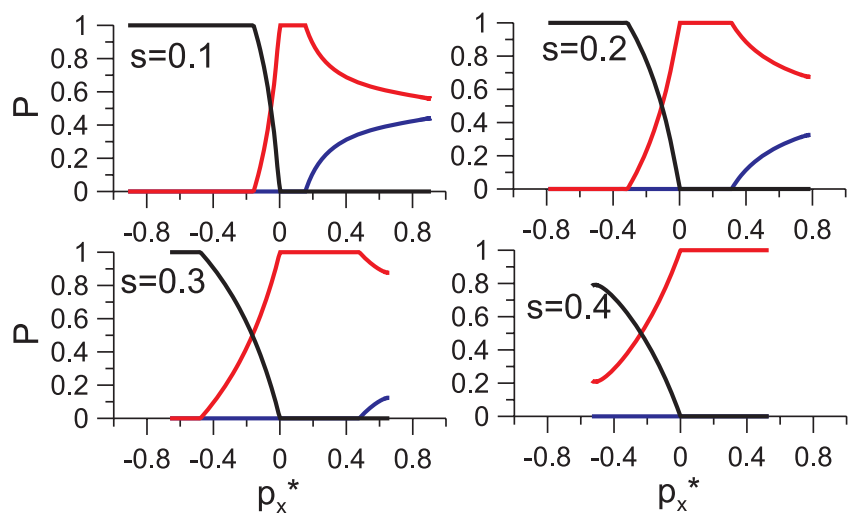

Fig. B1. Probabilities of capture $P_{1}$ (red), $P_{2}$ (blue) and $P=1-$ $P_{1}-P_{2}$ (black) are shown as functions of the parameter $p_{x}^{*}$ along the uncertainty curve for various $s$.

$(8 / 3)\left(1-s^{2}\right)^{3 / 2}$. As a result we have the following equation for $s_{\mathrm{bif}}$ :

$s_{\text {bif }}^{2 / 3}-s_{\text {bif }}^{2}=\left(\frac{2}{3 \pi}\right)^{2 / 3}\left(1-s_{\text {bif }}^{2}\right)$.

The solution of this equation is $s_{\mathrm{bif}} \approx 0.25$.

\section{Appendix B}

\section{Probabilities}

When trajectories reach the uncertainty curve, particles get from one potential well to another. If areas corresponding to both alternative wells are growing (i.e. corresponding $\Theta$ values are positive), particles choose one of the two accessible wells. The probability of being captured into one of the two small wells is $P_{1, \mathrm{r}}=\hat{\Theta}_{1, \mathrm{r}} /\left(\hat{\Theta}_{1}+\hat{\Theta}_{\mathrm{r}}+\hat{\Theta}\right)$, where $\hat{\Theta}_{1, \mathrm{r}}=\max \left(\Theta_{1, \mathrm{r}}, 0\right)$ and $\hat{\Theta}=\max \left(-\Theta_{1}-\Theta_{\mathrm{r}}, 0\right)$. The probability of being captured into the single well is $P=1-P_{1}-P_{\mathrm{r}}$ (see details in Neishtadt, 1987; Arnold et al., 2006).

For the system with $s=0$ we have $\Theta_{1}=\Theta_{\mathrm{r}}$ and $P_{1, \mathrm{r}}=1 / 2$ for $p_{x}^{*}>0$ (because there $\Theta_{1, \mathrm{r}}>0$ ), while $P_{1, \mathrm{r}}=0$ for $p_{x}^{*}<$ 0 . We plot probabilities $P_{1, \mathrm{r}}$ and $P$ for various values of the parameter $s$ in Fig. B1. As one can see, in systems with $s>$ 0.35 we have $P_{\mathrm{r}}=0$. Thus, in these systems particles cannot be captured into the right well at the uncertainty curve.

\section{Appendix C}

\section{Number of the uncertainty curve crossings}

In systems with $s<\bar{s}$, any trajectory can cross the uncertainty curve several times. However, for each particular value of $s$, the number of the uncertainty curve crossings is finite. To determine this number one can use the following scheme.
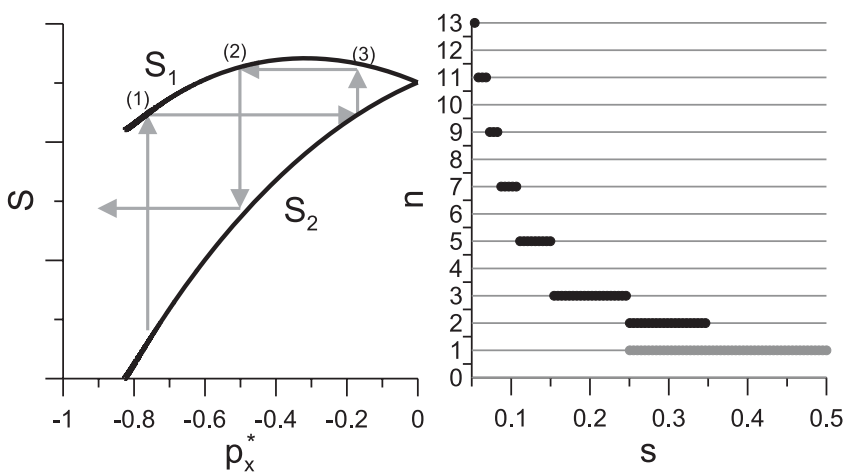

Fig. C1. Left panel demonstrates the scheme of the calculation of the number of uncertainty curve crossings. Right panel demonstrates the number of uncertainty curve crossings at $p_{x}^{*}<0$ for trajectories of the first type (black) and the second type (grey) as functions of $s$.

We plot profiles of $S_{1}$ and $S_{\mathrm{r}}$ for $p_{x}^{*}<0$. Then we plot the polygonal line with reflections from both curves $S_{\mathrm{l}, \mathrm{r}}$ until escape from the left side. This scheme is shown in the left panel of Fig. C1. In this scheme we use the same solid line for segments of graphs with positive and negative values of $\Theta_{1, \mathrm{r}}$. This scheme for any given initial value of $p_{x}^{*}$ at the uncertainty curve gives all possible values of $p_{x}^{*}<0$ at previous and subsequent arrivals to this curve. One can show that the corresponding adiabatic trajectory indeed passes through all these points. The number of reflection points in $S_{1}$ profile is equal to the number of the uncertainty curve crossings by trajectories of the first type at the half-space $p_{x}^{*}<0$ (the total number is twice larger). This number as a function of the parameter $s$ is shown in the right panel of Fig. C1 (black dots form a staircase-like graph). One can have arbitrary large number of the uncertainty curve crossings in the adiabatic approximation provided that $s$ is small enough. However, for very small $s$ dynamical jumps of the quasi-adiabatic invariant should be taken into account, see Appendix D.

Trajectories of the first type exist in the system with $s<\bar{s}$. With increase of the parameter $s$, the number of the uncertainty curve crossings decreases. The minimal number of the uncertainty curve crossings is four (two for the half-space $\left.p_{x}^{*}<0\right)$. Trajectories of the second type exist if $s_{\text {bif }}<s<1$. Trajectories of this type cross the uncertainty curve twice (once for the half-space $p_{x}^{*}<0$ ).

\section{Appendix D}

\section{Comparison of $\Delta I_{z}^{\text {geom }}$ and $\Delta I_{z}^{\text {dyn }}$}

For the current sheet with $s=0$, the dynamical jump of the quasi-adiabatic invariant is defined by the expression (Timofeev, 1978; Neishtadt, 1986; Cary et al., 1986; Neishtadt, 
1987; Büchner and Zelenyi, 1989):

$\Delta I_{z}^{\mathrm{dyn}}=-(2 / \pi) p_{x}^{*} \kappa \ln (2 \sin \pi \xi)$.

Here $\xi$ is the pseudo-phase. The value of $\xi$ characterizes fast variables at the separatrix crossing (it is treated as a random value uniformly distributed in the range $(0,1)$; see details in Neishtadt, 1986; Cary et al., 1986; Neishtadt, 1987; Arnold et al., 2006). As $\kappa \ll 1$, the dynamical jump can be considered as a small perturbation of the quasi-adiabatic invariant.

To compare $\Delta I_{z}^{\text {geom }}$ and $\Delta I_{z}^{\text {dyn }}$, we need to consider the system at small enough $s$. In this system we can expand the expressions for $p_{x}^{*}$ and $\kappa x^{*}$ (see Eq. A1):

$$
\begin{aligned}
& p_{x}^{*} \approx-\frac{z_{\mathrm{c}}}{s} A_{z_{\mathrm{c}}}+O\left(s^{2}\right) \\
& \kappa x^{*} \approx A_{z_{\mathrm{c}}}+O\left(s^{2}\right) .
\end{aligned}
$$

It is worth noting that in the principal approximation we have $\left(p_{x}^{*}\right)^{2}+\left(\kappa x^{*}\right)^{2}=1$. The corresponding expressions for $S_{\mathrm{l}, \mathrm{r}}$ can be written as

$S_{\mathrm{l}, \mathrm{r}}=\frac{8}{3}\left(\kappa x^{*}\right)^{3 / 2}+2 s\left(\frac{2 p_{x}^{*}}{\sqrt{\kappa x^{*}}} \pm \pi p_{x}^{*}\right)+O\left(s^{2}\right)$.

The geometrical jump is $\Delta I_{z}^{\text {geom }}=\left(S_{r}-S_{l}\right) / 2 \pi=-p_{x}^{*} s$. Therefore, we can conclude that for a system with $s \ll$ $(2 / \pi) \kappa \ln 2$ the geometrical jump is small enough. In this case one can consider only the dynamical jumps. Such a system is similar to one with $s=0$. In a system with $s \gg(2 / \pi) \kappa \ln 2$ the geometrical jumps dominate. The dynamical jumps produce slow diffusion across adiabatic trajectories.

Acknowledgements. We are very grateful to A. A. Vasiliev for useful discussion. This work was supported in part by the RF Presidential Program for the State Support of Leading Scientific Schools (project NSh-2519.2012.1) and the Russian Foundation for Basic Research (projects 11-02-01166, 12-02-91158).

Edited by: J. Büchner

Reviewed by: X. Zhou and one anonymous referee

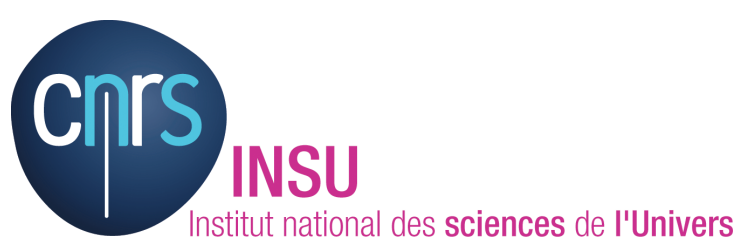

The publication of this article is financed by CNRS-INSU.

\section{References}

Anastasiadis, A., Gontikakis, C., and Efthymiopoulos, C.: Particle Interactions with Single or Multiple 3D Solar Reconnecting Current Sheets, Sol. Phys., 253, 199-214, doi:10.1007/s11207-0089234-4, 2008
Arnold, V. I.: Catastrophe Theory, Springer-Verlag, Berlin, 3rd Edn., 1992.

Arnold, V. I., Kozlov, V. V., and Neishtadt, A. I.: Mathematical Aspects of Classical and Celestial Mechanics, Dynamical Systems III. Encyclopedia of Mathematical Sciences, Springer-Verlag, New York, 3rd Edn., 2006.

Artemyev, A. V.: A model of one-dimensional current sheet with parallel currents and normal component of magnetic field, Phys. Plasmas, 18, 022104, doi:10.1063/1.3552141, 2011.

Artemyev, A. V., Neishtadt, A. I., and Zelenyi, L. M.: Jumps of adiabatic invariant at the separatrix of a degenerate saddle point, Chaos, 21, 043120, doi:10.1063/1.3657916, 2011.

Baek, S.-C., Choi, D.-I., and Horton, W.: Dawn-dusk magnetic field effects on ions accelerated in the current sheet, J. Geophys. Res., 100, 14935-14942, doi:10.1029/95JA01610, 1995.

Birn, J., Artemyev, A. V., Baker, D. N., Echim, M., Hoshino, M., and Zelenyi, L. M.: Particle acceleration in the magnetotail and aurora, Space Sci. Rev., 173, 49-102, 2012.

Büchner, J. and Zelenyi, L. M.: Deterministic chaos in the dynamics of charged particles near a magnetic field reversal, Phys. Lett. A, 118, 395-399, doi:10.1016/0375-9601(86)90268-9, 1986.

Büchner, J. and Zelenyi, L. M.: Regular and chaotic charged particle motion in magnetotaillike field reversals. I - Basic theory of trapped motion, J. Geophys. Res., 94, 11821-11842, doi:10.1029/JA094iA09p11821, 1989.

Büchner, J. and Zelenyi, L. M.: Regular and chaotic particle motion in sheared magnetic field reversals, Adv. Space Res., 11, 177182, doi:10.1016/0273-1177(91)90030-N, 1991.

Cary, J. R., Escande, D. F., and Tennyson, J. L.: Adiabatic-invariant change due to separatrix crossing, Phys. Rev. A, 34, 4256-4275, 1986.

Chapman, S. C. and Rowlands, G.: Are particles detrapped by constant $B_{y}$ in static magnetic reversals?, J. Geophys. Res., 103, 4597-4604, doi:10.1029/97JA01737, 1998.

Chen, J.: Nonlinear dynamics of charged particles in the magnetotail, J. Geophys. Res., 97, 15011-15050, doi:10.1029/92JA00955, 1992.

Chen, J. and Palmadesso, P. J.: Chaos and nonlinear dynamics of single-particle orbits in a magnetotaillike magnetic field, J. Geophys. Res., 91, 1499-1508, doi:10.1029/JA091iA02p01499, 1986.

Delcourt, D. C., Zelenyi, L. M., and Sauvaud, J.-A.: Magnetic moment scattering in a field reversal with nonzero $B_{y}$ component, J. Geophys. Res., 105, 349-360, doi:10.1029/1999JA900451, 2000.

Galeev, A. A. and Zelenyi, L. M.: Magnetic reconnection in a space plasma, in: Theoretical and Computational Plasma Physics, 93$116,1978$.

Galeev, A. A., Kuznetsova, M. M., and Zelenyi, L. M.: Magnetopause stability threshold for patchy reconnection, Space Sci. Rev., 44, 1-41, doi:10.1007/BF00227227, 1986.

Grigorenko, E. E., Zelenyi, L. M., Dolgonosov, M. S., Artemiev, A. V., Owen, C. J., Sauvaud, J.-A., Hoshino, M., and Hirai, M.: Non-adiabatic Ion Acceleration in the Earth Magnetotail and Its Various Manifestations in the Plasma Sheet Boundary Layer, Space Sci. Rev., 164, 133-181, doi:10.1007/s11214-011-9858-9, 2011.

Holland, D. L., Chen, J., and Agranov, A.: Effects of a constant cross-tail magnetic field on the particle dynamics 
in the magnetotail, J. Geophys. Res., 101, 24997-25002, doi:10.1029/95JA02282, 1996.

Karimabadi, H., Akimoto, K., Omidi, N., and Menyuk, C. R.: Particle acceleration by a wave in a strong magnetic field - Regular and stochastic motion, Phys. Fluids B-Plasma, 2, 606-628, doi:10.1063/1.859296, 1990.

Karimabadi, H., Daughton, W., and Quest, K. B.: Physics of saturation of collisionless tearing mode as a function of guide field, $\mathrm{J}$. Geophys. Res., 110, A03214, doi:10.1029/2004JA010749, 2005.

Kaufmann, R. L., Lu, C., and Larson, D. J.: Cross-tail current, fieldaligned current, and By, J. Geophys. Res., 99, 11277-11296, doi:10.1029/94JA00490, 1994.

Kuznetsova, M. M., Büchner, J., and Zelenyi, L. M.: Onset of collisionless magnetic reconnection in sheared field reversals, J. Geophys. Res., 101, 161-178, doi:10.1029/95JA02450, 1996.

Landau, L. D. and Lifshitz, E. M.: Vol. 1: Mechanics, Course of Theoretical Physics, Pergamon Press, 1st Edn., 1960.

Litvinenko, Y. E.: Regular Versus Chaotic Motion of Charged Particles in Non-Neutral Current Sheets, Sol. Phys., 147, 337-342, doi:10.1007/BF00690723, 1993.

Malova, H. V., Popov, V. Y., Mingalev, O. V., Mingalev, I. V., Mel'nik, M. N., Artemyev, A. V., Petrukovich, A. A., Delcourt, D. C., Shen, C., and Zelenyi, L. M.: Thin current sheets in the presence of a guiding magnetic field in Earth's magnetosphere, J. Geophys. Res., 117, A04212, doi:10.1029/2011JA017359, 2012.

Neishtadt, A. I.: Change of an adiabatic invariant at a separatrix, Soviet Journal of Plasma Physics, 12, 568-573, 1986.

Neishtadt, A. I.: On the change in the adiabatic invariant on crossing a separatrix in systems with two degrees of freedom, J. Appl. Math. Mech.-USS, 51, 586-592, doi:10.1016/00218928(87)90006-2, 1987.

Neishtadt, A. and Treschev, D.: Polymorphisms and adiabatic chaos, Ergod. Theor. Dyn. Syst., 31, 259-284, doi:10.1017/S0143385709001060, 2011.

Northrop, T. G.: The adiabatic motion of charged particles, Interscience Publishers John Wiley and Sons, New York-LondonSydney, 1963

Panov, E. V., Artemyev, A. V., Nakamura, R., and Baumjohann, W.: Two Types of Tangential Magnetopause Current Sheets: Cluster Observations and Theory, J. Geophys. Res., 116, A12204, doi:10.1029/2011JA016860, 2011.

Petrukovich, A. A.: Origins of plasma sheet $B_{y}$, J. Geophys. Res., 116, A07217, doi:10.1029/2010JA016386, 2011.

Schindler, K.: Adiabatic Particle Orbits in Discontinuous Fields, J. Math. Phys., 6, 313-321, doi:10.1063/1.1704282, 1965.

Sharma, A. S., Nakamura, R., Runov, A., Grigorenko, E. E., Hasegawa, H., Hoshino, M., Louarn, P., Owen, C. J., Petrukovich, A., Sauvaud, J.-A., Semenov, V. S., Sergeev, V. A., Slavin, J. A., Sonnerup, B. U. Ö., Zelenyi, L. M., Fruit, G., Haaland, S., Malova, H., and Snekvik, K.: Transient and localized processes in the magnetotail: a review, Ann. Geophys., 26, 9551006, doi:10.5194/angeo-26-955-2008, 2008.

Silin, I. and Büchner, J.: Nonlinear instability of thin current sheets in antiparallel and guided magnetic fields, Phys. Plasmas, 10, 3561-3570, doi:10.1063/1.1599357, 2003.

Silin, I. and Büchner, J.: Three-dimensional Vlasov-code simulations of magnetopause-like current sheets, Adv. Space Res., 37 , 1354-1362, doi:10.1016/j.asr.2005.05.025, 2006.
Sivukhin, D. V.: Motion of charged particles in electromagnetic fields in the drift approximation, vol. 1, 1-104, Consultants Bureau, New York, 1965.

Sonnerup, B. U. Ö.: Adiabatic particle orbits in a magnetic null sheet, J. Geophys. Res., 76, 8211-8222, doi:10.1029/JA076i034p08211, 1971.

Speiser, T. W.: Particle Trajectories in Model Current Sheets, 1, Analytical Solutions, J. Geophys. Res., 70, 4219-4226, doi:10.1029/JZ070i017p04219, 1965.

Speiser, T. W.: Particle Trajectories in Model Current Sheets, 2, Applications to Auroras Using a Geomagnetic Tail Model, J. Geophys. Res., 72, 3919-3932, doi:10.1029/JZ072i015p03919, 1967.

Timofeev, A. V.: On the problem of constancy of the adiabatic invariant during change of the type of motion, Sov. J. JETP, 75, 1303-1308, 1978.

Ukhorskiy, A. Y., Sitnov, M. I., Millan, R. M., and Kress, B. T.: The role of drift orbit bifurcations in energization and loss of electrons in the outer radiation belt, J. Geophys. Res., 116, A09208, doi:10.1029/2011JA016623, 2011.

Vainchtein, D. L., Büchner, J., Neishtadt, A. I., and Zelenyi, L. M.: Quasiadiabatic description of nonlinear particle dynamics in typical magnetotail configurations, Nonlin. Processes Geophys., 12, 101-115, doi:10.5194/npg-12-101-2005, 2005.

Vasiliev, A., Neishtadt, A., Artemyev, A., and Zelenyi, L.: Jump of the adiabatic invariant at a separatrix crossing: Degenerate cases, Physica D, 241, 566-573, doi:10.1016/j.physd.2011.11.015, 2012.

Whipple, E. C., Hill, J. R., and Nichols, J. D.: Magnetopause structure and the question of particle accessibility, J. Geophys. Res., 89, 1508-1516, doi:10.1029/JA089iA03p01508, 1984.

Whipple, E. C., Northrop, T. G., and Birmingham, T. J.: Adiabatic theory in regions of strong field gradients, J. Geophys. Res., 91, 4149-4156, doi:10.1029/JA091iA04p04149, 1986.

Wisdom, J.: A perturbative treatment of motion near the 3/1 commensurability, Icarus, 63, 272-289, doi:10.1016/00191035(85)90011-9, 1985.

Ynnerman, A., Chapman, S. C., Tsalas, M., and Rowlands, G.: Identification of symmetry breaking and a bifurcation sequence to chaos in single particle dynamics in magnetic reversals, Physica D, 139, 217-230, doi:10.1016/S0167-2789(99)00144$\mathrm{X}, 2000$.

Zelenyi, L. M. and Taktakishvili, A. L.: A kinetic theory of the magnetic islands merging instability, Plasma Phys. Contr. F., 30, 663679, doi:10.1088/0741-3335/30/6/003, 1988.

Zelenyi, L. M., Malova, H. V., Artemyev, A. V., Popov, V. Y., and Petrukovich, A. A.: Thin current sheets in collisionless plasma: Equilibrium structure, plasma instabilities, and particle acceleration, Plasma Phys. Rep., 37, 118-160, doi:10.1134/S1063780X1102005X, 2011.

Zhou, X.-Z., Pu, Z. Y., Zong, Q.-G., and Xie, L.: Energy filter effect for solar wind particle entry to the plasma sheet via flank regions during southward interplanetary magnetic field, J. Geophys. Res., 112, A06233, doi:10.1029/2006JA012180, 2007.

Zhu, Z. and Parks, G.: Particle orbits in model current sheet with a nonzero B(y) component, J. Geophys. Res., 98, 7603-7608, doi:10.1029/92JA02366, 1993. 